\title{
Article
}

\section{How Coffee Capsules Affect the Volatilome in Espresso Coffee}

\author{
Giuseppe Greco ${ }^{1}$, Estefanía Núñez-Carmona ${ }^{2, *} \mathbb{*}$, Marco Abbatangelo ${ }^{3}\left(\mathbb{D}\right.$, Patrizia Fava ${ }^{1}(\mathbb{D}$ \\ and Veronica Sberveglieri ${ }^{2,3}$ (I)
}

1 Department of Life Sciences, University of Modena and Reggio Emilia (UNIMORE), Via J.F.Kennedy 17/i, 42014 Reggio Emilia, Italy; giuseppegreco.2398@gmail.com (G.G.); patrizia.fava@unimore.it (P.F.)

2 National Research Council, Institute of Bioscience and Bioresources (CNR-IBBR), Via Madonna del Piano, 10, 50019 Sesto Fiorentino, Italy; veronica.sberveglieri@ibbr.cnr.it

3 Nano Sensor Systems, (NASYS) Spin-Off University of Brescia, Brescia, Via Camillo Brozzoni, 9, 25125 Brescia, Italy; marco.abbatangelo@nasys.it

* Correspondence: estefania.nunezcarmona@ibbr.cnr.it

check for

updates

Citation: Greco, G.;

Núñez-Carmona, E.; Abbatangelo, M.;

Fava, P.; Sberveglieri, V. How Coffee

Capsules Affect the Volatilome in

Espresso Coffee. Separations 2021, 8,

248. https://doi.org/10.3390/

separations 8120248

Academic Editors: Vlad Mureşan,

Cristina Anamaria Semeniuc and

Giorgia Purcaro

Received: 30 September 2021

Accepted: 13 December 2021

Published: 17 December 2021

Publisher's Note: MDPI stays neutral with regard to jurisdictional claims in published maps and institutional affiliations.

Copyright: (c) 2021 by the authors. Licensee MDPI, Basel, Switzerland. This article is an open access article distributed under the terms and conditions of the Creative Commons Attribution (CC BY) license (https:/ / creativecommons.org/licenses/by/ $4.0 /)$.

\begin{abstract}
Coffee capsules have become one of the most used methods to have a coffee in the last few years. In this work, coffee was prepared using a professional espresso coffee machine. We investigated the volatilome of four different polypropylene coffee capsule typologies (Biologico, Dolce, Deciso, Guatemala) with and without capsules in order to reveal the possible differences in the VOCs spectra. The volatilome of each one was singularly studied through an analysis by gas chromatography and mass spectrometry (GC-MS), checking the abundance of different VOCs in coffee extracted with and without a capsule protection and compared to its related sample. Furthermore, ANOVA and Tukey tests were applied to statistically identify and individuate the possible differences. As a result, it was found that coffee capsules, offer advantages of protecting coffee from oxidation or rancidity and, consequently extended shelf life as well as did not cause a reduction of volatile compounds intensity. Therefore, it is possible to conclude that the aroma of polypropylene coffee capsule extraction is not damaged compared to a traditional espresso.
\end{abstract}

Keywords: gas chromatography and mass spectrometry (GC-MS); volatilome; shot coffee; coffee capsules; aroma intensity; hydrogen

\section{Introduction}

The coffee capsule is a single-dose coffee product, which is roasted, ground and pressed perfectly portioned and inserted into a small, generally cylindrical aluminum or plastic container. The use of this kind of product has recently exponentially increased. The main reasons seem to be the rapidity and the good taste that characterize them, allowing them to be a real competitor of moka pot coffee by swaying consumer preferences. Indeed, its advantages would allow every Ho. Re. Ca. activity to serve to the consumer an instant and more aromatic product. Regarding to the shelf life of the portioned coffee, it is usually about 18 months or more [1] in relation to the materials and packaging techniques. Coffee has always been one of the most consumed beverages, the second in the world after water. Moreover, its consumption continues to increase. According to the International Coffee Organization (ICO) [2], the main intergovernmental organization for coffee, the best producers in the world are South American countries such as Brazil and Guatemala and Asian countries such as India and Vietnam, for Arabica and Robusta species, respectively. The two most preferred species that dominate the coffee market are Coffea arabica, produced in South America and East Africa, and Coffea canephora (Robusta), produced especially in Vietnam and Indonesia. The first one covers 70\% of the world's production, is more delicate, aromatic and slightly more acidic. The second most used one (30\%) is fragrant and with a stronger taste [3].

Coffee made with Arabica beans is more delicate but also more acid; its high presence of oils also makes it moderately bitter, especially due to polyphenols that are the main 
contributors to olive oil bitterness and astringency [4]. Coffee capsules can have several advantages; however, some drawbacks must be analyzed. The first drawback is undoubtedly the sustainability. The coffee capsule has a significant environmental impact due to difficulties on waste collection, treatment and disposal activities. Every year, the sale of coffee capsules reaches on average 10,000 billion units, producing around 120 thousand tons of waste, 70 of these only in Europe [5]. Another disadvantage is the safety of this product. Many theories affirm that there could be migration from the capsule materials to the packaged food [6,7]. It is well known how the consumer has recently started to pay more attention to the food safety and quality. This has happened because many people have discovered that a good diet and the assumption of certain compounds, as the antioxidants can be found in different foods, can be preventive against heart and carcinogenic diseases [6]. On the contrary, some compounds that can migrate from packaging to packaged food can be harmful and as a result a new consumer can be reluctant to use coffee capsules. Thus, the same packaging, which represents an advantage for better maintenance and its protection capacities in favor of the food inside including it organoleptic and aromatic characteristics, has a flip side. This work focuses on the fraction volatile of coffee capsule samples. The "term volatilome" or the synonym volatome [8] has been used since the beginning of the decade of 2010. The volatilome refers to all the volatile metabolites as well as other volatile organic and inorganic compounds that originate from a natural or artificial matrix, as for example, food, organisms or even an ecosystem. It can be considered that all the metabolites in the volatilome can be classified and as a subset of the metabolome but also contains exogenously derived compounds that do not derive from metabolic processes. Therefore, the volatilome is considered a distinct entity from the metabolome.

This work aims to analyze if coffee aroma intensity can be influenced by the performance of the capsules. Particularly, if the intensity of each volatile compound could be higher due to the compression for which the capsule is subject during the extraction. The investigation of the volatilome was carried out through gas chromatography and mass spectrometry (GC-MS) analysis [9], checking the abundance of different components in coffee extracted with and without a capsule protection. Furthermore, statistical data analysis was performed in particular univariate ANOVA and Tukey tests and multivariate statistics as PCA (principal component analysis.

\section{Materials and Methods}

\subsection{Sample Description and Preparation}

The considered samples are four typologies of coffee capsule: "Biologico", "Deciso", "Dolce" and "Guatemala", gently supplied by "Caffè Molinari" (Via Francia, Modena, Italy). The "Spaziale S2" (LA SPAZIALE S.p.A., Casalecchio di Reno, Italia), professional machine was used to extract coffee. The coffee extraction occurred using [10] $18 \mathrm{~L}$ containers of "Rocca Galgana" (Citerna di Fornovo di Taro, Parma, Italy) mineral water to overcome the possibility of having inhomogeneities due to the water. This study was conducted in two phases: during the first phase, coffee was prepared simply by entering the coffee capsule into the correspondent extraction tool; in the second phase, coffee was pulled out of each capsule, it was manually pressed in the group, and extracted without capsule. Three replicates for each sample were carried out, obtaining 12 vials of coffee with capsule and 12 vials of those without capsule. The same quantity of coffee $(62.5 \mathrm{~mL}$, espresso shot coffee [11]) was extracted and collected using a beaker. After, $20 \mathrm{~mL}$ chromatographic vials were used, each filled with $1.2 \mathrm{~mL}$ of coffee to proceed with the analysis. The vials containing the sample were sealed by aluminum rings and a septa made of polytetrafluoroethylene (PTFE) and silicone. The vial containing the sample, before analysis, was placed in the incubator, where it was initially incubated at $70^{\circ} \mathrm{C}$ for $10 \mathrm{~min}$, in order to heat the sample and achieve the equilibrium of the volatile compounds between the headspace and the liquid phase and to remove any variables. After, VOCs extraction was performed using solid phase microextraction (SPME) technique. The fiber used for the adsorption of volatile compounds was a divinylbenzene/carboxen/polydimethylsiloxane (DVB/CAR/PDMS) 
50/30 $\mu \mathrm{m}$ (Supelco Co. Bellefonte, PA, USA). It was exposed in the head space of the vial for $20 \mathrm{~min}$ at $70{ }^{\circ} \mathrm{C}$, to favor the absorption of the volatile compounds.

\subsection{GC-MS Analysis Conditions}

The GC instrument used in this work was a Shimadzu GC 2010 PLUS (Kyoto, Japan), equipped with a Shimadzu single quadrupole mass spectrometer (MS) MS-QP2020 Ultra (Kyoto, Japan). The fiber was inserted into the injector of the GC-MS [12], at a temperature of $240{ }^{\circ} \mathrm{C}$ for $6 \mathrm{~min}$, to allow the desorption of the molecules constituting the volatilome. The desorption occurred through a standard methodic. It started with a temperature of $40{ }^{\circ} \mathrm{C}$ for $5 \mathrm{~min}$, then followed with an increase of $3{ }^{\circ} \mathrm{C} /$ minute until reaching $180{ }^{\circ} \mathrm{C}$. Once reached $180^{\circ} \mathrm{C}$ the temperature undergoes a further rise, this time of $10^{\circ} \mathrm{C} /$ minute, until reaching $240{ }^{\circ} \mathrm{C}$, which are maintained for five minutes, for a total program time of $62 \mathrm{~min}$ [13]. GC was operated in the direct mode throughout the run, which means that all VOCs desorbed inside the injector goes inside the column. The separation was performed on a MEGA-5MS capillary column, $25 \mathrm{~m} \times 0.25 \mathrm{~mm} \times 0.25 \mu \mathrm{m}$ film thickness, (Agilent Technologies, Santa Clara, CA, USA). Hydrogen was used as the carrier gas and was produced by GENius PF500, FullTech Instruments Srl (Rome, Italy) at a constant flow rate of $2.34 \mathrm{~mL} / \mathrm{min}$. The interface connecting the GC to the MS was kept at $240{ }^{\circ} \mathrm{C}$, the MS was maintained in $\mathrm{El}$ mode at $70 \mathrm{ev}$ and the ion source at $240{ }^{\circ} \mathrm{C}$. Mass spectra were recorded in a range of 35 to $500 \mathrm{~m} / \mathrm{z}$ in TIC mode (total ion count) with scanning intervals at $0.3 \mathrm{~s}$ [14-16]. Once the sample was analyzed, chromatograms with the peaks corresponding to the VOCs were obtained. The chromatographic peaks obtained were integrated in automatic mode using, as a parameter, the peak area, considering at least 80 peaks and with an area value not less than $500 \mathrm{AMU}$. Identification of the compounds was made using NIST11 and FFNSC2 mass spectra libraries. Other parameters used in the automatic peak integration were slope $100 / \mathrm{min}$, width $2 \mathrm{~s}$, drift $0 / \mathrm{min}$, and doubling time (T.DBL) $1000 \mathrm{~min}$, and no smoothing method was applied.

\subsection{GC-MS Data Analysis}

The abundance [17] of each volatile compound is the average of the abundance of the three replicates for each sample. In addition, the standard deviation was also calculated in order to give a better vision of the results. This output was validated using the statistical analysis of ANOVA test to see if our results have statistical significance with a $p>0.05$ In addition, Tukey's test was applied in order to verify which samples statistically were different between each other. In addition, multivariate analysis was performed through PCA investigation using the abundance means for each compound as a feature. Data analysis was performed using MATLAB ${ }^{\circledR}$ R2015a software (MathWorks, Natick, MA, USA).

\section{Results and Discussion}

Regarding the data extracted from the GC-MS-SPME analysis [18], the volatile fingerprints for each coffee sample were identified in Table 1. The latter shows the abundance average of the three replicates for each common compound in every typology. Figures 1-4 show the comparison of the volatilome intensity, by means of abundance, of coffee extract with and without capsule. It is possible to observe that in general for the four typologies, the abundance of the compounds in the samples with capsule is higher compared to the one without capsule. This is highlighted in the graphs, thus the coffee aroma intensity [19] can be influenced by the performance of the capsule. According to GC-MS analysis, the capsule could determine a difference of intensity for some components and, consequently, to obtain a different and more fragrant product compared to coffee obtained without the capsule. Coffee volatilome is one of the most complex in nature regarding the food matrix. 


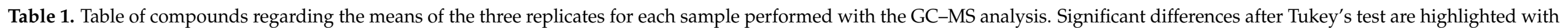

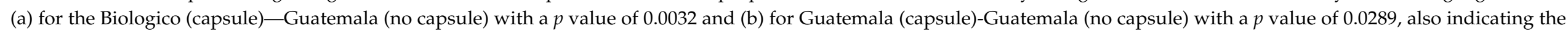

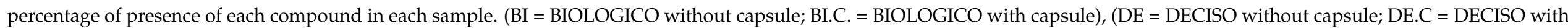
capsule), (DO = DOLCE without capsule; DO.C. = DOLCE with capsule) and (GU = GUATEMALA without capsule; GU.C = GUATEMALA with capsule).

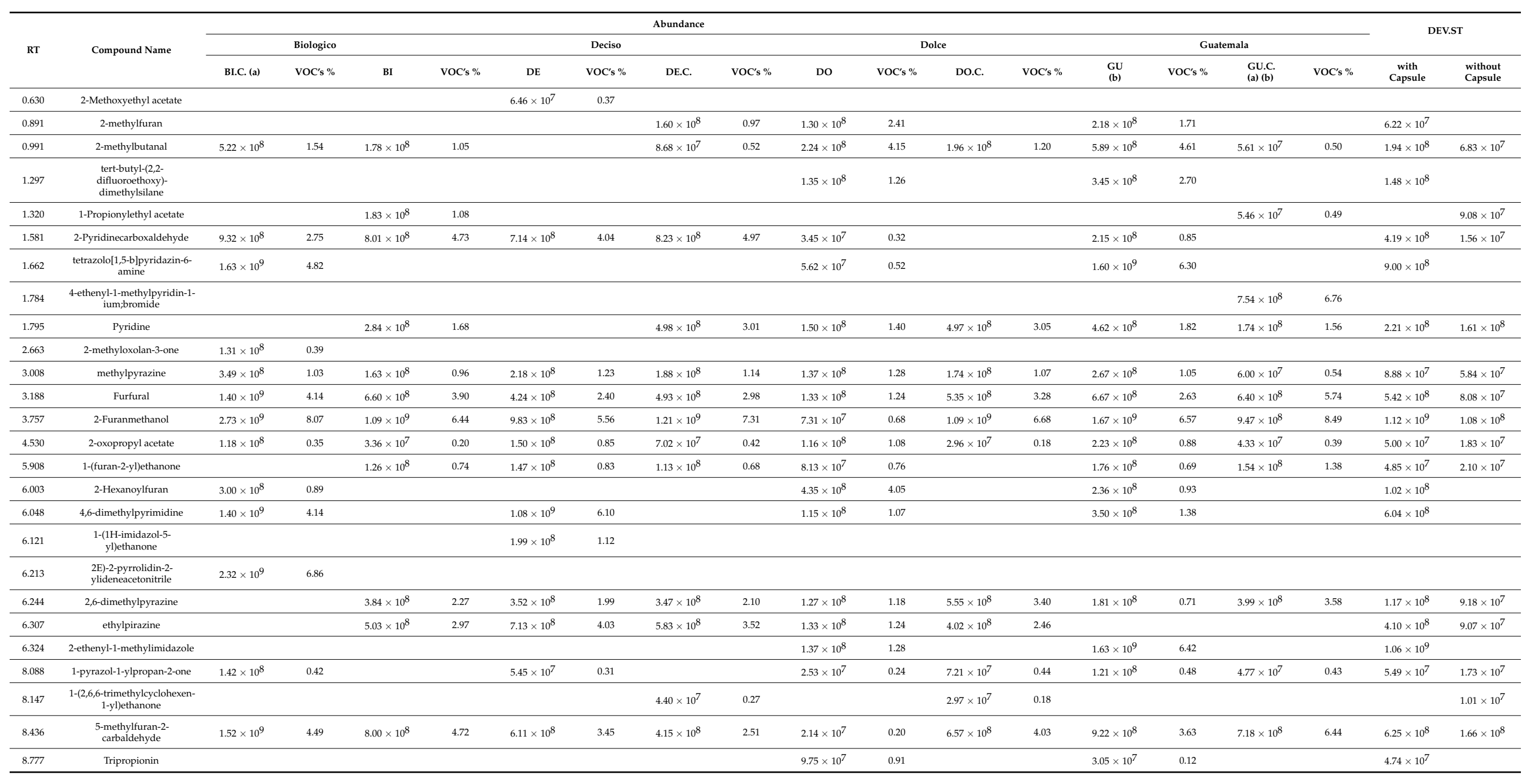


Table 1. Cont

\begin{tabular}{|c|c|c|c|c|c|c|c|c|c|c|c|c|c|c|c|c|c|c|c|}
\hline 8.845 & 2-methyl,3-Pentanone & $7.74 \times 10^{8}$ & 2.29 & & & & & & & & & & & & & & & & \\
\hline 8.873 & Ethyl propionate & $3.45 \times 10^{8}$ & 1.02 & $7.13 \times 10^{7}$ & 0.42 & & & & & $1.28 \times 10^{8}$ & 1.19 & & & $3.94 \times 10^{8}$ & 1.55 & $1.43 \times 10^{8}$ & 1.28 & $1.42 \times 10^{8}$ & $5.07 \times 10^{7}$ \\
\hline 8.883 & 3,3-dimethylbutan-2-one & & & & & & & & & & & $2.52 \times 10^{7}$ & 0.15 & & & $7.17 \times 10^{7}$ & 0.64 & & $3.29 \times 10^{7}$ \\
\hline 9.027 & 2-oxobutyl acetate & & & $6.76 \times 10^{7}$ & 0.40 & & & & & & & & & & & $6.90 \times 10^{7}$ & 0.62 & & $9.90 \times 10^{5}$ \\
\hline 9.757 & Phenol & $1.84 \times 10^{8}$ & 0.54 & & & $1.69 \times 10^{8}$ & 0.96 & $1.75 \times 10^{8}$ & 1.06 & $5.50 \times 10^{7}$ & 0.51 & $1.46 \times 10^{8}$ & 0.89 & $1.89 \times 10^{8}$ & 0.74 & $1.34 \times 10^{8}$ & 1.20 & $6.34 \times 10^{7}$ & $2.11 \times 10^{7}$ \\
\hline 9.852 & $\begin{array}{c}(4- \\
\text { hydroxyphenly)phosphonic } \\
\text { acid }\end{array}$ & & & $8.32 \times 10^{7}$ & 0.49 & & & & & & & & & & & & & & \\
\hline 9.979 & furan-2-ylmethyl acetate & $2.35 \times 10^{9}$ & 6.95 & $7.53 \times 10^{8}$ & 4.45 & $8.67 \times 10^{8}$ & 4.90 & $1.28 \times 10^{9}$ & 7.73 & $6.27 \times 10^{7}$ & 0.58 & $9.66 \times 10^{8}$ & 5.92 & $1.14 \times 10^{9}$ & 4.49 & $7.95 \times 10^{8}$ & 7.13 & $9.48 \times 10^{8}$ & $2.39 \times 10^{8}$ \\
\hline 10.283 & furan-2-ylmethylurea & $1.77 \times 10^{9}$ & 5.23 & $1.63 \times 10^{9}$ & 9.62 & $1.85 \times 10^{9}$ & 10.46 & $1.43 \times 10^{9}$ & 8.64 & $5.00 \times 10^{7}$ & 0.47 & $1.51 \times 10^{9}$ & 9.25 & $1.42 \times 10^{9}$ & 5.59 & $1.19 \times 10^{9}$ & 10.67 & $8.36 \times 10^{8}$ & $1.86 \times 10^{8}$ \\
\hline 10.479 & $\begin{array}{c}\text { 1-methylpyrrole-2- } \\
\text { carbaldehyde }\end{array}$ & & & $7.66 \times 10^{8}$ & 4.52 & $8.61 \times 10^{8}$ & 4.87 & $7.79 \times 10^{8}$ & 4.71 & $5.60 \times 10^{7}$ & 0.52 & $9.91 \times 10^{8}$ & 6.07 & $9.07 \times 10^{8}$ & 3.57 & $3.71 \times 10^{8}$ & 3.33 & $4.79 \times 10^{8}$ & $2.59 \times 10^{8}$ \\
\hline 10.524 & 2-Ethyl-5-methylpyrazine & & & $6.10 \times 10^{8}$ & 3.60 & & & $4.28 \times 10^{8}$ & 2.59 & & & $5.45 \times 10^{8}$ & 3.34 & & & & & & $9.22 \times 10^{7}$ \\
\hline 10.567 & 2-methoxy-3H-azepine & $1.09 \times 10^{9}$ & 3.22 & & & $8.13 \times 10^{8}$ & 4.60 & & & & & & & & & & & $1.96 \times 10^{8}$ & \\
\hline 10.578 & $\begin{array}{l}\text { 4-(2-bromoethyl)-3,5- } \\
\text { dimethyl-1H-pyrazole }\end{array}$ & & & & & & & $7.10 \times 10^{8}$ & 4.29 & & & & & & & $4.02 \times 10^{8}$ & 3.60 & & $2.18 \times 10^{8}$ \\
\hline 10.601 & $\begin{array}{l}\text { 2-chloro-1-(1-methylpyrrol-2- } \\
\text { yl)ethanone }\end{array}$ & & & & & & & & & $1.90 \times 10^{7}$ & 0.18 & & & $1.48 \times 10^{9}$ & 5.83 & & & $1.03 \times 10^{9}$ & \\
\hline 10.742 & 2-propanoyl furan & $2.99 \times 10^{8}$ & 0.88 & $1.44 \times 10^{8}$ & 0.85 & $1.02 \times 10^{8}$ & 0.58 & $8.63 \times 10^{7}$ & 0.52 & & & & & & & $8.79 \times 10^{7}$ & 0.79 & $1.39 \times 10^{8}$ & $3.29 \times 10^{7}$ \\
\hline 10.883 & 2-propylpyrazine & & & & & $6.99 \times 10^{7}$ & 0.40 & & & & & & & & & & & & \\
\hline 11.163 & 2-ethyl-6-methylpyrazine & & & & & & & & & $7.40 \times 10^{7}$ & 0.69 & & & $1.04 \times 10^{8}$ & 0.41 & & & $2.12 \times 10^{7}$ & \\
\hline 11.211 & 2-prop-1-en-2-ylpyrazine & $1.16 \times 10^{8}$ & 0.34 & $3.65 \times 10^{7}$ & 0.22 & & & $3.67 \times 10^{7}$ & 0.22 & & & $3.98 \times 10^{7}$ & 0.24 & & & & & & $1.85 \times 10^{6}$ \\
\hline 11.255 & $\begin{array}{l}\text { 1-(1-methoxypropan-2- } \\
\text { yloxy)propan-2-ol }\end{array}$ & & & $4.62 \times 10^{7}$ & 0.27 & & & & & & & & & & & & & & \\
\hline 11.439 & 1-(4H-pyridin-1-yl)ethanone & & & & & & & & & $5.45 \times 10^{7}$ & 0.51 & & & & & & & & \\
\hline 11.478 & $\begin{array}{l}\text { 5-amino-3-methyl-1,2- } \\
\text { oxazole-4-carbonitrile }\end{array}$ & $3.95 \times 10^{8}$ & 1.17 & & & & & & & & & & & & & & & & \\
\hline 11.494 & 1-ethyl pyrrole & & & $1.31 \times 10^{8}$ & 0.77 & $1.13 \times 10^{8}$ & 0.64 & $1.69 \times 10^{8}$ & 1.02 & & & $1.91 \times 10^{8}$ & 1.17 & $1.05 \times 10^{8}$ & 0.41 & & & $5.66 \times 10^{6}$ & $3.04 \times 10^{7}$ \\
\hline 11.522 & 2-methoxybenzenamine & $3.17 \times 10^{8}$ & 0.94 & & & & & & & $1.15 \times 10^{8}$ & 1.07 & & & $3.27 \times 10^{8}$ & 1.29 & & & $1.20 \times 10^{8}$ & \\
\hline 11.533 & 1-(4H-pyridin-1-y) ethanone & & & & & & & & & & & & & $2.18 \times 10^{8}$ & 0.86 & & & & \\
\hline 11.560 & $N$-(2-Cyanoethyl)-pyrrole & $2.81 \times 10^{8}$ & 0.83 & & & & & & & & & & & & & & & & \\
\hline 11.563 & 1H-pyrrole-2-carbaldehyde & $3.29 \times 10^{8}$ & 0.97 & & & & & & & $8.61 \times 10^{8}$ & 8.02 & & & $2.43 \times 10^{8}$ & 0.96 & $1.24 \times 10^{8}$ & 1.11 & $3.35 \times 10^{8}$ & \\
\hline 11.673 & $\begin{array}{l}\text { 3-methylcyclopentane-1,2- } \\
\text { dione }\end{array}$ & & & & & & & & & & & & & & & $2.58 \times 10^{7}$ & 0.23 & & \\
\hline 11.700 & $\begin{array}{c}\text { 2-hydroxy-3- } \\
\text { methylcyclopent-2-en-1-one }\end{array}$ & & & $1.77 \times 10^{7}$ & 0.10 & & & $1.72 \times 10^{7}$ & 0.10 & & & $2.23 \times 10^{7}$ & 0.14 & & & $2.60 \times 10^{7}$ & 0.23 & & $4.16 \times 10^{7}$ \\
\hline 12.011 & 2-ethylhexanol & & & & & & & & & & & & & & & $5.02 \times 10^{7}$ & 0.45 & & \\
\hline 12.025 & $\begin{array}{l}\text { 1-(2-methylcyclopenten-1- } \\
\text { yl)ethanone }\end{array}$ & $2.24 \times 10^{8}$ & 0.66 & $8.38 \times 10^{7}$ & 0.49 & $8.77 \times 10^{7}$ & 0.50 & $8.30 \times 10^{7}$ & 0.50 & $8.56 \times 10^{7}$ & 0.80 & $5.59 \times 10^{7}$ & 0.34 & $1.34 \times 10^{8}$ & 0.53 & $7.61 \times 10^{7}$ & 0.68 & $6.48 \times 10^{7}$ & $1.30 \times 10^{7}$ \\
\hline
\end{tabular}


Table 1. Cont.

\begin{tabular}{|c|c|c|c|c|c|c|c|c|c|c|c|c|c|c|c|c|c|c|c|}
\hline 12.083 & $\begin{array}{l}\text { (4E)-2,6-dimethylhepta-2,4- } \\
\text { diene }\end{array}$ & & & & & $6.03 \times 10^{7}$ & 0.34 & & & & & & & $1.37 \times 10^{8}$ & 0.54 & & & $5.42 \times 10^{7}$ & \\
\hline 12.093 & 3,3,5-trimethylcyclohexene & & & & & & & $5.93 \times 10^{7}$ & 0.36 & & & $4.76 \times 10^{7}$ & 0.29 & & & $7.67 \times 10^{7}$ & 0.69 & & $1.46 \times 10^{7}$ \\
\hline 12.133 & 6,6-Dimethylhepta-2,4-diene & & & & & & & & & & & & & & & $4.03 \times 10^{7}$ & 0.36 & & \\
\hline 12.603 & Benzeneacetaldehyde & & & & & & & & & & & $2.56 \times 10^{7}$ & 0.16 & & & $2.32 \times 10^{7}$ & 0.21 & & $1.70 \times 10^{6}$ \\
\hline 12.623 & Phenylacetaldehyde & $4.28 \times 10^{7}$ & 0.13 & $2.23 \times 10^{7}$ & 0.13 & $3.46 \times 10^{7}$ & 0.20 & $3.94 \times 10^{7}$ & 0.24 & $2.19 \times 10^{8}$ & 2.04 & $1.83 \times 10^{7}$ & 0.11 & $4.21 \times 10^{7}$ & 0.17 & $2.65 \times 10^{7}$ & 0.24 & $8.97 \times 10^{7}$ & $9.15 \times 10^{6}$ \\
\hline 12.675 & $\begin{array}{c}N, N-\text { dimethyl-1-(5- } \\
\text { methylfuran-2- } \\
\text { yl)methanamine }\end{array}$ & $1.17 \times 10^{8}$ & 0.35 & & & & & & & $3.64 \times 10^{8}$ & 3.39 & & & $8.90 \times 10^{7}$ & 0.35 & $4.27 \times 10^{7}$ & 0.38 & $1.51 \times 10^{8}$ & \\
\hline 12.715 & $\begin{array}{l}\text { 1,4-dimethyl-5-propan-2- } \\
\text { ylcyclopentene }\end{array}$ & $3.62 \times 10^{8}$ & 1.07 & & & & & & & & & & & & & & & & \\
\hline 12.737 & $\begin{array}{l}\text { 1-(1-ethylpyrazol-4- } \\
\text { yl)ethanone }\end{array}$ & $3.34 \times 10^{7}$ & 0.10 & & & & & $4.20 \times 10^{7}$ & 0.25 & & & & & & & & & & \\
\hline 12.771 & $\begin{array}{c}\text { 5-methyl-2- } \\
\text { Furanmethanethiol }\end{array}$ & & & & & & & & & & & & & & & $4.24 \times 10^{7}$ & 0.38 & & \\
\hline 13.018 & 1-(furan-2-yl)butan-2-one & & & $2.03 \times 10^{7}$ & 0.12 & & & $2.15 \times 10^{7}$ & 0.13 & & & $1.96 \times 10^{7}$ & 0.12 & & & $1.77 \times 10^{7}$ & 0.16 & & $1.59 \times 10^{6}$ \\
\hline 13.266 & $\begin{array}{l}\text { 2,3,4-trimethylycylopent-2- } \\
\text { en-1-one }\end{array}$ & & & & & $4.80 \times 10^{7}$ & 0.27 & & & & & & & & & & & & \\
\hline 13.290 & 5-ethylfuran-2-carbaldehyde & $9.11 \times 10^{7}$ & 0.27 & & & & & & & $4.65 \times 10^{7}$ & 0.43 & & & $4.98 \times 10^{7}$ & 0.20 & & & $2.49 \times 10^{7}$ & \\
\hline 13.334 & 2-methylphenol & $8.35 \times 10^{7}$ & 0.25 & $5.66 \times 10^{7}$ & 0.33 & $4.39 \times 10^{7}$ & 0.25 & $5.32 \times 10^{7}$ & 0.32 & $2.84 \times 10^{7}$ & 0.26 & $5.46 \times 10^{7}$ & 0.33 & $5.37 \times 10^{7}$ & 0.21 & $5.35 \times 10^{7}$ & 0.48 & $2.32 \times 10^{7}$ & $1.54 \times 10^{6}$ \\
\hline 13.597 & 1-phenylethanone & & & & & $4.93 \times 10^{7}$ & 0.28 & & & & & & & & & & & & \\
\hline 13.760 & 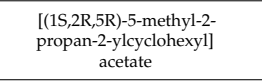 & $1.66 \times 10^{8}$ & 0.49 & & & & & & & & & & & & & & & & \\
\hline 13.823 & Linalool oxide <cis-> & & & $5.93 \times 10^{7}$ & 0.35 & $7.74 \times 10^{7}$ & 0.44 & $5.49 \times 10^{7}$ & 0.33 & $2.46 \times 10^{7}$ & 0.23 & & & $7.07 \times 10^{7}$ & 0.28 & & & $2.87 \times 10^{7}$ & $3.11 \times 10^{6}$ \\
\hline 14.045 & 2-methoxybenzenamine & & & $3.76 \times 10^{8}$ & 2.22 & & & $3.03 \times 10^{8}$ & 1.83 & & & & & & & & & & $5.16 \times 10^{7}$ \\
\hline 14.054 & 1-(1H-pyrrol-2-yl)ethanone & $6.30 \times 10^{8}$ & 1.86 & $1.97 \times 10^{8}$ & 1.16 & $4.45 \times 10^{8}$ & 2.52 & $3.34 \times 10^{8}$ & 2.02 & $3.39 \times 10^{7}$ & 0.32 & $1.35 \times 10^{8}$ & 0.83 & $5.01 \times 10^{8}$ & 1.97 & $8.05 \times 10^{7}$ & 0.72 & $2.58 \times 10^{8}$ & $1.09 \times 10^{8}$ \\
\hline 14.159 & $\begin{array}{c}\begin{array}{c}\text { 3-ethyl-2,5- } \\
\text { dimethylpyrazine }\end{array} \\
\end{array}$ & $1.12 \times 10^{9}$ & 3.31 & $4.09 \times 10^{8}$ & 2.42 & $6.62 \times 10^{8}$ & 3.74 & $3.97 \times 10^{8}$ & 2.40 & $1.37 \times 10^{8}$ & 1.28 & $6.97 \times 10^{8}$ & 4.27 & $7.88 \times 10^{8}$ & 3.10 & $1.91 \times 10^{8}$ & 1.71 & $4.08 \times 10^{8}$ & $2.08 \times 10^{8}$ \\
\hline 14.185 & 3,5-Dimethyl-4-allylpyrazole & $1.59 \times 10^{9}$ & 4.70 & & & & & & & & & & & & & & & & \\
\hline 14.373 & 2-(furan-2-ylmethyl)furan & $2.38 \times 10^{8}$ & 0.70 & $1.38 \times 10^{8}$ & 0.81 & $2.69 \times 10^{8}$ & 1.52 & $1.67 \times 10^{8}$ & 1.01 & $2.50 \times 10^{7}$ & 0.23 & $1.04 \times 10^{8}$ & 0.64 & $1.52 \times 10^{8}$ & 0.60 & $1.13 \times 10^{8}$ & 1.01 & $1.09 \times 10^{8}$ & $2.83 \times 10^{7}$ \\
\hline 14.566 & 2-methoxyphenol & $1.03 \times 10^{9}$ & 3.04 & $4.30 \times 10^{8}$ & 2.54 & $8.89 \times 10^{8}$ & 5.02 & $5.01 \times 10^{8}$ & 3.03 & $4.22 \times 10^{7}$ & 0.39 & $5.24 \times 10^{8}$ & 3.21 & $7.99 \times 10^{8}$ & 3.15 & $1.46 \times 10^{8}$ & 1.31 & $4.42 \times 10^{8}$ & $1.74 \times 10^{8}$ \\
\hline 14.612 & 4-methoxyphenol & & & & & & & & & & & $3.72 \times 10^{8}$ & 2.28 & & & $2.30 \times 10^{8}$ & 2.06 & & $1.00 \times 10^{8}$ \\
\hline 14.737 & $\begin{array}{l}\text { (2,6-dimethylcyclohexen-1- } \\
\text { yl } \\
\text { actate }\end{array}$ & & & $1.00 \times 10^{8}$ & 0.59 & & & $1.08 \times 10^{8}$ & 0.65 & $4.06 \times 10^{7}$ & 0.38 & & & $1.27 \times 10^{8}$ & 0.50 & & & $6.11 \times 10^{7}$ & $5.66 \times 10^{6}$ \\
\hline 14.751 & $\begin{array}{l}\text { 3-methylcyclohexane-1,2- } \\
\text { dione }\end{array}$ & & & & & & & & & & & & & & & $8.34 \times 10^{7}$ & 0.75 & & \\
\hline 14.867 & furan-2-ylmethyl propanoate & & & $2.03 \times 10^{8}$ & 1.20 & $3.21 \times 10^{8}$ & 1.81 & $2.31 \times 10^{8}$ & 1.40 & $2.76 \times 10^{7}$ & 0.26 & $2.05 \times 10^{8}$ & 1.26 & $2.60 \times 10^{8}$ & 1.02 & $1.20 \times 10^{8}$ & 1.08 & $1.55 \times 10^{8}$ & $4.82 \times 10^{7}$ \\
\hline 15.000 & 4,5-dimethylhex-4-en-3-one & & & & & & & & & & & & & & & $4.29 \times 10^{7}$ & 0.38 & & \\
\hline
\end{tabular}


Table 1. Cont.

\begin{tabular}{|c|c|c|c|c|c|c|c|c|c|c|c|c|c|c|c|c|c|c|c|}
\hline 15.246 & $\begin{array}{l}\text { 2-methylpropyl } \\
{[(Z) \text {-non-3-enyl] carbonate }}\end{array}$ & & & & & $2.22 \times 10^{8}$ & 1.25 & & & & & $8.30 \times 10^{7}$ & 0.51 & & & $1.47 \times 10^{8}$ & 1.32 & & $4.53 \times 10^{7}$ \\
\hline 15.246 & $\begin{array}{c}\text { Glutraic acid, } \\
\text { di(cis-non-3-enyl) ester }\end{array}$ & $2.43 \times 10^{8}$ & 0.72 & & & & & & & & & $1.64 \times 10^{8}$ & 1.01 & & & & & & \\
\hline 15.255 & (4Z)-cyclooct-4-en-1-one & & & $7.30 \times 10^{7}$ & 0.43 & & & & & & & & & & & & & & \\
\hline 15.268 & $\begin{array}{c}\text { 1,1-Dimethyl-4- } \\
\text { methylenecyclohexane }\end{array}$ & & & & & & & & & & & & & & & $5.59 \times 10^{7}$ & 0.50 & & \\
\hline 15.291 & $\begin{array}{l}\text { 4-O-[(Z)-non-3-enyl] } \\
\text { 1-O-propyy } \\
\text { (E)-but-2-enedioate }\end{array}$ & & & & & $2.58 \times 10^{8}$ & 1.46 & & & & & & & & & & & & \\
\hline 15.293 & $\begin{array}{l}\text { 3-methylidene-3a,4,5,6,7,7a- } \\
\text { hexahydro-1-benzofuran-2- } \\
\text { one }\end{array}$ & & & & & & & & & & & $7.13 \times 10^{7}$ & 0.44 & & & & & & \\
\hline 15.318 & $\begin{array}{l}\text { 3,7-dimethylocta-1,-dien-3- } \\
\text { ol }\end{array}$ & $2.05 \times 10^{8}$ & 0.61 & & & & & & & & & & & & & & & & \\
\hline 15.449 & 3,7-dimethyloctan-3-ol & & & $6.34 \times 10^{7}$ & 0.37 & & & & & & & & & & & & & & \\
\hline 15.459 & 2-prop-1-en-2-ylpyrazine & & & & & & & & & & & & & & & $4.31 \times 10^{7}$ & 0.39 & & \\
\hline 15.702 & Nonanal & $1.76 \times 10^{8}$ & 0.52 & & & & & & & & & & & & & & & & \\
\hline 15.723 & $\begin{array}{c}\text { 2-(1-methylpyrrol-2- } \\
\text { yl)acetonitrile }\end{array}$ & & & $2.47 \times 10^{8}$ & 1.46 & & & $3.66 \times 10^{8}$ & 2.21 & $3.31 \times 10^{7}$ & 0.31 & $3.20 \times 10^{8}$ & 1.96 & $1.74 \times 10^{8}$ & 0.69 & $1.91 \times 10^{8}$ & 1.71 & $9.96 \times 10^{7}$ & $7.75 \times 10^{7}$ \\
\hline 15.759 & $\begin{array}{l}\text { 1-cyclopentylethyl } \\
\text { 5-chloropentanoate }\end{array}$ & & & & & $1.21 \times 10^{8}$ & 0.68 & & & & & & & & & & & & \\
\hline 15.794 & $\begin{array}{l}\text { 3-hydroxy-2-methylpyran-4- } \\
\text { one }\end{array}$ & $1.31 \times 10^{8}$ & 0.39 & & & & & & & & & & & & & & & & \\
\hline 15.963 & $\begin{array}{l}\text { 2,5-Methano-1H-indene, } \\
\text { octahydro- }\end{array}$ & & & $4.27 \times 10^{7}$ & 0.25 & & & & & & & & & & & $4.77 \times 10^{7}$ & 0.43 & & $3.54 \times 10^{6}$ \\
\hline 16.061 & $\begin{array}{l}\text { 1-(3-methylpyrazin-2- } \\
\text { yl)ethanone }\end{array}$ & $2.61 \times 10^{8}$ & 0.77 & & & & & & & & & & & & & & & & \\
\hline 16.107 & $\begin{array}{l}\text { 3-ethyl-2-hydroxycyclopent- } \\
\text { 2-en-1-one }\end{array}$ & & & $1.35 \times 10^{8}$ & 0.80 & & & $1.09 \times 10^{8}$ & 0.66 & & & $1.39 \times 10^{8}$ & 0.85 & & & & & & $1.63 \times 10^{7}$ \\
\hline 16.131 & ethyl non-2-enoate & $5.95 \times 10^{7}$ & 0.18 & $7.13 \times 10^{7}$ & 0.42 & & & $1.10 \times 10^{8}$ & 0.66 & & & & & & & $6.62 \times 10^{7}$ & 0.59 & & $2.40 \times 10^{7}$ \\
\hline 16.138 & $\begin{array}{l}\text { 3-butylcyclopentane-1,2,- } \\
\text { trione }\end{array}$ & & & $6.40 \times 10^{7}$ & 0.38 & & & & & & & & & & & & & & \\
\hline 16.167 & $\begin{array}{c}\text { 1-(6-methylpyrazin-2- } \\
\text { yl)ethanone }\end{array}$ & & & & & & & & & $4.41 \times 10^{7}$ & 0.41 & $5.07 \times 10^{7}$ & 0.31 & $1.30 \times 10^{8}$ & 0.51 & & & $6.07 \times 10^{7}$ & \\
\hline 16.381 & $\begin{array}{c}\text { prop-2-enyl } \\
\text { furan-2-carboxylate }\end{array}$ & $3.12 \times 10^{8}$ & 0.92 & $7.67 \times 10^{7}$ & 0.45 & & & & & $1.60 \times 10^{7}$ & 0.15 & $9.93 \times 10^{7}$ & 0.61 & $2.24 \times 10^{8}$ & 0.88 & $6.53 \times 10^{7}$ & 0.59 & $1.52 \times 10^{8}$ & $1.73 \times 10^{7}$ \\
\hline 16.540 & $\begin{array}{l}N-[(4-\text { fluorophenyl)methyl]- } \\
\text { 2-phenylethanamine }\end{array}$ & $1.48 \times 10^{7}$ & 0.04 & & & & & & & & & & & & & & & & \\
\hline 16.753 & $\begin{array}{c}\text { 1-(5-methylfuran-2- } \\
\text { yl)propan-2-one }\end{array}$ & $2.06 \times 10^{8}$ & 0.61 & & & & & & & & & & & & & & & & \\
\hline 16.774 & $\begin{array}{l}\text { (2E,4E)-2,4-dimethylhepta- } \\
\text { 2,-dienal }\end{array}$ & $1.93 \times 10^{8}$ & 0.57 & $6.77 \times 10^{7}$ & 0.40 & $1.52 \times 10^{8}$ & 0.86 & $8.82 \times 10^{7}$ & 0.53 & $1.86 \times 10^{7}$ & 0.17 & $9.79 \times 10^{7}$ & 0.60 & $1.35 \times 10^{8}$ & 0.53 & $4.83 \times 10^{7}$ & 0.43 & $7.48 \times 10^{7}$ & $2.21 \times 10^{7}$ \\
\hline 16.795 & $\begin{array}{l}\text { 2-methyl-3-(2- } \\
\text { methylpropyl)pyrazine }\end{array}$ & & & & & & & $9.70 \times 10^{7}$ & 0.59 & & & & & & & & & & \\
\hline
\end{tabular}


Table 1. Cont.

\begin{tabular}{|c|c|c|c|c|c|c|c|c|c|c|c|c|c|c|c|c|c|c|c|}
\hline 17.097 & $\begin{array}{l}\text { 1-(-4-methoxyphenyl)-2-(6- } \\
\text { methylpyrazin-2-yl)ethanol }\end{array}$ & & & $7.89 \times 10^{7}$ & 0.47 & & & $6.58 \times 10^{7}$ & 0.40 & & & $5.70 \times 10^{7}$ & 0.35 & & & & & & $1.10 \times 10^{7}$ \\
\hline 17.120 & $\begin{array}{l}\text { 5-methyl-6, } 7 \text {-dihydro-5H- } \\
\text { cyclopenta[blbyrazine }\end{array}$ & & & $7.02 \times 10^{7}$ & 0.41 & & & & & & & & & & & & & & \\
\hline 17.216 & $\begin{array}{l}\text { 4-(5-methylfuran-2-y)butan- } \\
\text { 2-one }\end{array}$ & & & & & & & $1.03 \times 10^{8}$ & 0.62 & & & & & & & & & & \\
\hline 17.550 & adamantan-1-ol & & & & & & & & & $5.16 \times 10^{8}$ & 4.80 & & & $3.45 \times 10^{7}$ & 0.14 & & & $3.40 \times 10^{8}$ & \\
\hline 17.572 & $\begin{array}{l}1,3,5 \text {-trimethylpiperidin-4- } \\
\text { one }\end{array}$ & $1.32 \times 10^{8}$ & 0.39 & $6.51 \times 10^{7}$ & 0.38 & & & $3.89 \times 10^{7}$ & 0.24 & $1.30 \times 10^{8}$ & 1.21 & $7.21 \times 10^{7}$ & 0.44 & $5.62 \times 10^{7}$ & 0.22 & & & $4.32 \times 10^{7}$ & $1.75 \times 10^{7}$ \\
\hline 17.900 & $\begin{array}{l}\text { 3,4-dimethyl-1H-pyrrole-2- } \\
\text { carbaldehyde }\end{array}$ & & & $1.38 \times 10^{8}$ & 0.81 & $1.39 \times 10^{8}$ & 0.79 & $9.25 \times 10^{7}$ & 0.56 & $1.35 \times 10^{8}$ & 1.26 & $2.05 \times 10^{8}$ & 1.26 & $1.37 \times 10^{8}$ & 0.54 & $5.44 \times 10^{7}$ & 0.49 & $2.00 \times 10^{6}$ & $6.48 \times 10^{7}$ \\
\hline 17.946 & $\begin{array}{l}\text { 3,5-diethyl-2- } \\
\text { methylpyrazine }\end{array}$ & & & & & & & & & & & & & $1.50 \times 10^{8}$ & 0.59 & & & & \\
\hline 17.990 & $\begin{array}{c}\text { 2-ethyl-3,5,6- } \\
\text { trimethylpyrazine }\end{array}$ & $6.11 \times 10^{8}$ & 1.81 & $1.07 \times 10^{8}$ & 0.63 & & & $9.15 \times 10^{7}$ & 0.55 & $3.45 \times 10^{7}$ & 0.32 & $8.16 \times 10^{7}$ & 0.50 & $1.33 \times 10^{8}$ & 0.52 & $4.51 \times 10^{7}$ & 0.40 & $3.08 \times 10^{8}$ & $2.63 \times 10^{7}$ \\
\hline 18.123 & $\begin{array}{c}\mathrm{N}-(2- \\
\text { methoxyphyl)formamide } \\
\end{array}$ & $8.68 \times 10^{7}$ & 0.26 & & & & & & & $5.62 \times 10^{7}$ & 0.52 & $3.88 \times 10^{7}$ & 0.24 & $7.31 \times 10^{7}$ & 0.29 & & & $1.53 \times 10^{7}$ & \\
\hline 18.291 & $\begin{array}{c}\text { 2,3-diethyl-5- } \\
\text { methylpyrazine }\end{array}$ & $1.34 \times 10^{8}$ & 0.40 & $6.23 \times 10^{7}$ & 0.37 & & & $7.27 \times 10^{7}$ & 0.44 & $1.50 \times 10^{8}$ & 1.40 & $6.89 \times 10^{7}$ & 0.42 & $1.16 \times 10^{8}$ & 0.46 & $5.81 \times 10^{7}$ & 0.52 & $1.70 \times 10^{7}$ & $6.54 \times 10^{6}$ \\
\hline 18.511 & $\begin{array}{l}\text { 2,5-dimethyl-3-propan-2- } \\
\text { ylpyrazine }\end{array}$ & $1.40 \times 10^{8}$ & 0.41 & & & & & & & $1.37 \times 10^{8}$ & 1.28 & & & $8.13 \times 10^{7}$ & 0.32 & & & $3.31 \times 10^{7}$ & \\
\hline 18.530 & $\begin{array}{c}1-(3-\text { ethylpyrazin-2- } \\
\text { yl)ethanone }\end{array}$ & & & & & & & & & & & $9.88 \times 10^{7}$ & 0.61 & & & & & & \\
\hline 18.730 & 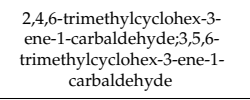 & & & & & $1.15 \times 10^{8}$ & 0.65 & $3.15 \times 10^{7}$ & 0.19 & & & & & & & & & & \\
\hline 18.830 & 2-n-Heptylfuran & $1.38 \times 10^{8}$ & 0.41 & $1.02 \times 10^{8}$ & 0.60 & & & $7.41 \times 10^{7}$ & 0.45 & $1.33 \times 10^{8}$ & 1.24 & $6.96 \times 10^{7}$ & 0.43 & $1.15 \times 10^{8}$ & 0.45 & & & $1.21 \times 10^{7}$ & $1.76 \times 10^{7}$ \\
\hline 18.841 & $\begin{array}{l}\text { (5R)-5-methyl-2-propan-2- } \\
\text { ylidenecyclohexan-1-one }\end{array}$ & & & & & & & & & & & & & & & $7.22 \times 10^{7}$ & 0.65 & & \\
\hline 18.850 & $\begin{array}{c}\text { 2-(2, } 2,6,6 \text {-rimethylycyclohexen- } \\
\text { 1-yl)acetaldehyde }\end{array}$ & $1.42 \times 10^{8}$ & 0.42 & & & & & & & & & & & & & & & & \\
\hline 18.868 & 1-(furan-2-yl)propan-2-one & $1.47 \times 10^{8}$ & 0.43 & & & & & & & & & & & & & & & & \\
\hline 19.013 & 1-(furan-2-ylmethyl)pyrrole & & & $1.07 \times 10^{8}$ & 0.63 & & & & & & & & & & & $5.04 \times 10^{7}$ & 0.45 & & $4.00 \times 10^{7}$ \\
\hline 19.192 & 5-methoxybenzene-1,3-diol & $4.53 \times 10^{8}$ & 1.34 & $2.99 \times 10^{8}$ & 1.77 & $3.43 \times 10^{8}$ & 1.94 & $3.79 \times 10^{8}$ & 2.29 & $7.31 \times 10^{7}$ & 0.68 & $4.14 \times 10^{8}$ & 2.54 & $4.35 \times 10^{8}$ & 1.71 & $2.23 \times 10^{8}$ & 2.00 & $1.75 \times 10^{8}$ & $8.54 \times 10^{7}$ \\
\hline 19.456 & $\begin{array}{c}1,2,3,4,4 \mathrm{4a}, 5,6,7,8,8 \mathrm{a}- \\
\text { decahydronaphthalene }\end{array}$ & & & $1.59 \times 10^{8}$ & 0.94 & & & $1.36 \times 10^{8}$ & 0.82 & $1.16 \times 10^{8}$ & 1.08 & $9.82 \times 10^{7}$ & 0.60 & $1.33 \times 10^{8}$ & 0.52 & & & $1.20 \times 10^{7}$ & $3.07 \times 10^{7}$ \\
\hline 19.468 & $\begin{array}{c}1,3,3 \mathrm{a}, 4,6,6 \mathrm{6a}- \\
\text { hexahydropentalene-2,5- } \\
\text { dione }\end{array}$ & & & & & $9.82 \times 10^{7}$ & 0.56 & & & & & & & & & & & & \\
\hline 19.478 & $\begin{array}{c}N-(4- \\
\text { hydroxyphenyl)acetamide } \\
\end{array}$ & & & & & & & $8.44 \times 10^{7}$ & 0.51 & & & & & & & & & & \\
\hline 19.569 & $\begin{array}{c}N-(3- \\
\text { hydroxyphenyl)acetamide }\end{array}$ & & & & & & & & & & & & & & & $7.04 \times 10^{7}$ & 0.63 & & \\
\hline 19.600 & $\begin{array}{l}\text { (Z)-3-(furan-2-yl)-2- } \\
\text { methylprop-2-enal }\end{array}$ & & & & & & & & & & & & & & & $7.49 \times 10^{7}$ & 0.67 & & \\
\hline
\end{tabular}


Table 1. Cont.

\begin{tabular}{|c|c|c|c|c|c|c|c|c|c|c|c|c|c|c|c|c|c|c|c|}
\hline 19.723 & $\begin{array}{l}\text { 2-methyl-5-[(E)-prop-1- } \\
\text { enyl]pyrazine }\end{array}$ & $7.42 \times 10^{7}$ & 0.22 & & & & & & & & & & & & & $5.14 \times 10^{7}$ & 0.46 & & \\
\hline 19.896 & $\begin{array}{c}5,6,7,8- \\
\text { tetrahydroquinoxaline }\end{array}$ & $1.91 \times 10^{8}$ & 0.56 & $7.94 \times 10^{7}$ & 0.47 & $1.57 \times 10^{8}$ & 0.89 & $9.04 \times 10^{7}$ & 0.55 & $8.13 \times 10^{7}$ & 0.76 & $1.21 \times 10^{8}$ & 0.74 & $1.27 \times 10^{8}$ & 0.50 & & & $4.66 \times 10^{7}$ & $2.16 \times 10^{7}$ \\
\hline 19.947 & $\begin{array}{l}2,5 \text {-dimethyl-3- } \\
\text { propylpyrazine }\end{array}$ & & & & & & & & & $1.15 \times 10^{8}$ & 1.07 & & & $1.37 \times 10^{8}$ & 0.54 & & & $1.56 \times 10^{7}$ & \\
\hline 20.150 & $\begin{array}{l}\text { 1-(3,6-dimethylpyrazin-2-yl)- } \\
\text { 3-methylbutan-1-one }\end{array}$ & & & & & & & & & $4.35 \times 10^{8}$ & 4.05 & & & $2.53 \times 10^{7}$ & 0.10 & & & $2.90 \times 10^{8}$ & \\
\hline 20.163 & benzene-1,2-diol & & & & & $4.02 \times 10^{7}$ & 0.23 & & & & & & & & & & & & \\
\hline 20.362 & 2,5-dimethylthiophene & $1.21 \times 10^{8}$ & 0.36 & & & & & $6.01 \times 10^{7}$ & 0.36 & $1.27 \times 10^{8}$ & 1.18 & $1.04 \times 10^{8}$ & 0.64 & $9.75 \times 10^{7}$ & 0.38 & & & $1.56 \times 10^{7}$ & $3.10 \times 10^{7}$ \\
\hline 20.413 & 1-hydroxypyridin-2-imine & & & & & & & & & & & $2.97 \times 10^{7}$ & 0.18 & & & & & & \\
\hline 20.419 & $\begin{array}{c}\text { (4-hexadecanoyloxy-5- } \\
\text { hydroxy-6-menthylpyridin-3- } \\
\text { yll } \\
\text { hexadecanoate }\end{array}$ & & & & & & & & & $1.37 \times 10^{8}$ & 1.28 & & & $1.28 \times 10^{8}$ & 0.50 & & & $6.36 \times 10^{6}$ & \\
\hline 20.460 & Decanal & & & $2.71 \times 10^{7}$ & 0.16 & & & & & & & & & & & & & & \\
\hline 20.623 & Undecanal & & & $7.48 \times 10^{7}$ & 0.44 & & & $6.26 \times 10^{7}$ & 0.38 & $2.53 \times 10^{7}$ & 0.24 & $4.62 \times 10^{7}$ & 0.28 & $1.01 \times 10^{8}$ & 0.40 & $3.49 \times 10^{7}$ & 0.31 & $5.35 \times 10^{7}$ & $1.76 \times 10^{7}$ \\
\hline 20.638 & $\begin{array}{l}\text { 3,7-dimethyl-6,7-dihydro- } \\
\text { 5H-cyclopenta[b]pyrazine }\end{array}$ & & & & & $5.39 \times 10^{7}$ & 0.30 & & & $2.14 \times 10^{7}$ & 0.20 & $3.71 \times 10^{7}$ & 0.23 & $5.50 \times 10^{7}$ & 0.22 & & & $1.91 \times 10^{7}$ & \\
\hline 21.129 & Tridecanedial & $5.78 \times 10^{7}$ & 0.17 & $2.52 \times 10^{7}$ & 0.15 & $2.93 \times 10^{7}$ & 0.17 & $2.27 \times 10^{7}$ & 0.14 & $1.28 \times 10^{8}$ & 1.19 & $5.19 \times 10^{7}$ & 0.32 & $5.00 \times 10^{7}$ & 0.20 & & & $4.29 \times 10^{7}$ & $1.62 \times 10^{7}$ \\
\hline 22.372 & $\begin{array}{l}\text { 2-methoxy-3-propan-2- } \\
\text { ylpyrazine }\end{array}$ & & & & & $1.44 \times 10^{7}$ & 0.08 & & & & & & & & & & & & \\
\hline 22.427 & 2--soamyl-6-methylpyrazine & & & & & & & & & $6.27 \times 10^{7}$ & 0.58 & & & $7.40 \times 10^{7}$ & 0.29 & & & $7.99 \times 10^{6}$ & \\
\hline 22.427 & $\begin{array}{l}\text { 3H-benzimidazole-5- } \\
\text { carbaldehyde }\end{array}$ & $6.53 \times 10^{7}$ & 0.19 & $3.25 \times 10^{7}$ & 0.19 & $3.50 \times 10^{7}$ & 0.20 & $2.56 \times 10^{7}$ & 0.15 & $5.00 \times 10^{7}$ & 0.47 & $2.94 \times 10^{7}$ & 0.18 & $1.90 \times 10^{7}$ & 0.07 & & & $1.99 \times 10^{7}$ & $3.46 \times 10^{6}$ \\
\hline 22.841 & 3H-quinazolin-4-one & & & $3.99 \times 10^{7}$ & 0.24 & & & $6.46 \times 10^{7}$ & 0.39 & & & & & & & & & & $1.75 \times 10^{7}$ \\
\hline 22.879 & heptadecan-9-one & & & & & $1.17 \times 10^{8}$ & 0.66 & & & & & & & & & & & & \\
\hline 23.208 & 8-hydroxyneomenthol & & & $2.60 \times 10^{7}$ & 0.15 & & & & & & & & & & & & & & \\
\hline 23.236 & $\begin{array}{c}\text { 2-[(furan-2- } \\
\text { ylmethyldisulfanyl)methyl]ffuran }\end{array}$ & & & $4.15 \times 10^{7}$ & 0.25 & & & & & & & & & & & & & & \\
\hline 23.239 & 1,2-dibromocyclohexane & & & $9.36 \times 10^{7}$ & 0.55 & & & & & & & & & & & $5.15 \times 10^{7}$ & 0.46 & & $2.98 \times 10^{7}$ \\
\hline 23.279 & $\begin{array}{l}\text { 1,4-dimethoxy-2-methyl-5- } \\
\text { propylbenzzene }\end{array}$ & & & & & & & & & & & $5.50 \times 10^{7}$ & 0.34 & & & & & & \\
\hline 23.339 & $\begin{array}{c}\text { 1-(-4-propan-2- } \\
\text { ylphenyl)ethanone }\end{array}$ & & & & & & & & & & & & & & & $3.81 \times 10^{7}$ & 0.34 & & \\
\hline 23.342 & $\begin{array}{c}\text { 1-(2,5- } \\
\text { dihydroxyphenyl)ethanone }\end{array}$ & $5.92 \times 10^{7}$ & 0.17 & $4.62 \times 10^{7}$ & 0.27 & & & & & & & $3.81 \times 10^{7}$ & 0.23 & & & & & & $5.73 \times 10^{6}$ \\
\hline 23.350 & $\begin{array}{l}\text { 1-(2,4-dihydroxyphenyl)-2- } \\
\text { phenylethanone }\end{array}$ & $1.30 \times 10^{8}$ & 0.38 & $6.21 \times 10^{7}$ & 0.37 & $9.45 \times 10^{7}$ & 0.53 & $5.68 \times 10^{7}$ & 0.34 & $5.60 \times 10^{7}$ & 0.52 & & & $1.15 \times 10^{8}$ & 0.45 & & & $3.21 \times 10^{7}$ & $3.75 \times 10^{6}$ \\
\hline 23.359 & 2-acetylresorcinol & & & $8.44 \times 10^{7}$ & 0.50 & & & & & & & & & & & $5.09 \times 10^{7}$ & 0.46 & & $2.37 \times 10^{7}$ \\
\hline
\end{tabular}


Table 1. Cont.

\begin{tabular}{|c|c|c|c|c|c|c|c|c|c|c|c|c|c|c|c|c|c|c|c|}
\hline 23.363 & 4-ethyl-2-methoxyphenol & & & & & & & & & & & & & $4.77 \times 10^{7}$ & 0.19 & & & & \\
\hline 23.518 & $\begin{array}{l}\text { 1,2-dimethoxy-3- } \\
\text { methylbenzene }\end{array}$ & & & & & & & & & & & & & $2.38 \times 10^{8}$ & 0.94 & & & & \\
\hline 23.534 & $\begin{array}{l}\text { 4-(2-aminoethyl)-2- } \\
\text { methoxyphenol }\end{array}$ & & & & & & & & & & & & & $2.42 \times 10^{8}$ & 0.95 & & & & \\
\hline 23.538 & decan-1-ol & & & $4.04 \times 10^{8}$ & 2.39 & & & & & $5.17 \times 10^{8}$ & 4.81 & & & & & & & & \\
\hline 23.543 & Nonanoic acid & $8.23 \times 10^{8}$ & 2.43 & $7.93 \times 10^{8}$ & 4.68 & $8.62 \times 10^{8}$ & 4.87 & $8.06 \times 10^{8}$ & 4.87 & $1.90 \times 10^{7}$ & 0.18 & $9.42 \times 10^{8}$ & 5.77 & $8.61 \times 10^{8}$ & 3.39 & & & $4.15 \times 10^{8}$ & $8.25 \times 10^{7}$ \\
\hline 23.733 & Benzo-2,3-pyrrole & & & $2.28 \times 10^{7}$ & 0.13 & & & & & & & & & & & & & & \\
\hline 24.081 & $\begin{array}{l}\text { 1-(5-methylfuran-2- } \\
\text { yl)propan-2-one }\end{array}$ & & & & & & & & & & & & & & & $3.94 \times 10^{7}$ & 0.35 & & \\
\hline 24.334 & $\begin{array}{l}\text { 1-fluoro-2-[(2-methylpropan- } \\
\text { 2-yl)oxymethyllbenzzene }\end{array}$ & $1.17 \times 10^{8}$ & 0.35 & & & & & & & & & & & & & & & & \\
\hline 24.363 & adamantane-1,2-diamine & $9.38 \times 10^{7}$ & 0.28 & & & $7.52 \times 10^{7}$ & 0.43 & $8.75 \times 10^{7}$ & 0.53 & $7.40 \times 10^{7}$ & 0.69 & $8.75 \times 10^{7}$ & 0.54 & $8.56 \times 10^{7}$ & 0.34 & $2.58 \times 10^{7}$ & 0.23 & $9.35 \times 10^{6}$ & $3.56 \times 10^{7}$ \\
\hline 24.501 & $\begin{array}{c}\text { 2-(furan-2- } \\
\text { ylmethoxymethyl)furan }\end{array}$ & & & $1.76 \times 10^{7}$ & 0.10 & & & & & & & & & & & $2.37 \times 10^{7}$ & 0.21 & & $4.31 \times 10^{6}$ \\
\hline 24.503 & 4-ethenyl-2-methoxyphenol & & & & & & & & & & & & & & & $1.87 \times 10^{7}$ & 0.17 & & \\
\hline 24.546 & $\begin{array}{c}\text { 2,3-dimethyl-5-(3- } \\
\text { methylbutyl)pyrazine }\end{array}$ & & & & & & & & & & & & & & & $3.14 \times 10^{7}$ & 0.28 & & \\
\hline 24.762 & $\begin{array}{c}\text { 2,5-dimethyl-3-(3-- } \\
\text { methylbutyl)pyrazine }\end{array}$ & & & $1.54 \times 10^{8}$ & 0.91 & $2.91 \times 10^{8}$ & 1.64 & $2.13 \times 10^{8}$ & 1.29 & $5.45 \times 10^{7}$ & 0.51 & $1.43 \times 10^{8}$ & 0.88 & $2.19 \times 10^{8}$ & 0.86 & $1.25 \times 10^{8}$ & 1.12 & $1.21 \times 10^{8}$ & $3.81 \times 10^{7}$ \\
\hline 25.204 & $\begin{array}{l}\text { 4-ethyl-1,2- } \\
\text { dimethoxybenzene }\end{array}$ & & & $1.61 \times 10^{8}$ & 0.95 & $1.51 \times 10^{8}$ & 0.85 & & & & & $2.03 \times 10^{8}$ & 1.24 & & & $8.61 \times 10^{7}$ & 0.77 & & $5.92 \times 10^{7}$ \\
\hline 25.221 & $\begin{array}{l}2,4,4 \text {-trimethyl-3-(3-- } \\
\text { methylbutyl)cyclohex-2-en- } \\
\text { 1-one }\end{array}$ & & & $4.30 \times 10^{8}$ & 2.54 & & & $2.84 \times 10^{8}$ & 1.72 & $1.15 \times 10^{8}$ & 1.07 & $5.22 \times 10^{8}$ & 3.20 & $3.64 \times 10^{8}$ & 1.43 & & & $1.76 \times 10^{8}$ & $1.20 \times 10^{8}$ \\
\hline 25.350 & 2-aminonaphthalen-1-ol & $3.79 \times 10^{7}$ & 0.11 & & & & & & & $8.61 \times 10^{8}$ & 8.02 & & & $4.65 \times 10^{7}$ & 0.18 & & & $4.73 \times 10^{8}$ & \\
\hline 25.383 & 4-methyl-1H-quinolin-2-one & $4.00 \times 10^{7}$ & 0.12 & $2.70 \times 10^{7}$ & 0.16 & $4.01 \times 10^{7}$ & 0.23 & $1.72 \times 10^{7}$ & 0.10 & & & $2.28 \times 10^{7}$ & 0.14 & & & & & $7.07 \times 10^{4}$ & $4.92 \times 10^{6}$ \\
\hline 25.870 & 2-methylchromen-4-one & $2.54 \times 10^{7}$ & 0.08 & $1.17 \times 10^{7}$ & 0.07 & $6.85 \times 10^{7}$ & 0.39 & $3.74 \times 10^{7}$ & 0.23 & $8.56 \times 10^{7}$ & 0.80 & $2.62 \times 10^{7}$ & 0.16 & $2.84 \times 10^{7}$ & 0.11 & $1.42 \times 10^{7}$ & 0.13 & $2.98 \times 10^{7}$ & $1.18 \times 10^{7}$ \\
\hline 26.529 & $\begin{array}{l}\text { 4-ethenyl-1,2- } \\
\text { dimethoxybenzene }\end{array}$ & $3.54 \times 10^{7}$ & 0.10 & & & & & & & & & & & & & & & & \\
\hline 26.737 & $\begin{array}{l}\text { 2-methoxy-5-(E)-prop-1- } \\
\text { enyl]phenol }\end{array}$ & & & & & $2.57 \times 10^{7}$ & 0.15 & $1.63 \times 10^{7}$ & 0.10 & $2.19 \times 10^{8}$ & 2.04 & & & $2.31 \times 10^{7}$ & 0.09 & & & $1.12 \times 10^{8}$ & \\
\hline 26.761 & $\begin{array}{c}\text { 4-ethoxy-3- } \\
\text { hydroxybenzaldehyde }\end{array}$ & $1.19 \times 10^{8}$ & 0.35 & & & $2.92 \times 10^{7}$ & 0.17 & & & $3.64 \times 10^{8}$ & 3.39 & & & $2.46 \times 10^{7}$ & 0.10 & & & $1.59 \times 10^{8}$ & \\
\hline 26.904 & $\begin{array}{l}\text { 2-methoxy-4-prop-2- } \\
\text { enylphenol }\end{array}$ & & & $3.67 \times 10^{7}$ & 0.22 & $7.34 \times 10^{7}$ & 0.41 & & & & & & & & & $1.85 \times 10^{7}$ & 0.17 & & $1.29 \times 10^{7}$ \\
\hline 27.813 & $\begin{array}{c}\text { 7-methyl-4-oxo-1H-1,8- } \\
\text { naphthyridine-3-carboxylic } \\
\text { acid }\end{array}$ & & & $3.39 \times 10^{7}$ & 0.20 & & & & & & & & & & & & & & \\
\hline 27.817 & $\begin{array}{l}\text { 1-(2,6,6-trimethylcyclohexa- } \\
1,3-\text {-dien-1-y)but-2-en-1-one }\end{array}$ & & & $2.07 \times 10^{7}$ & 0.12 & & & $1.83 \times 10^{7}$ & 0.11 & $4.65 \times 10^{7}$ & 0.43 & $1.50 \times 10^{7}$ & 0.09 & $3.39 \times 10^{7}$ & 0.13 & & & $8.91 \times 10^{6}$ & $2.86 \times 10^{6}$ \\
\hline 27.825 & 3-methyl-1H-indole & & & & & $5.83 \times 10^{7}$ & 0.33 & & & & & & & & & & & & \\
\hline 27.842 & dodecan-2-ol & $3.97 \times 10^{7}$ & 0.12 & & & & & & & & & & & & & & & & \\
\hline 28.016 & Tetradecane & & & & & & & & & $2.84 \times 10^{7}$ & 0.26 & & & $1.37 \times 10^{8}$ & 0.54 & & & $7.68 \times 10^{7}$ & \\
\hline 28.057 & $\begin{array}{l}5 \text {-(1,5-dimethylpyrrol-2-yl)- } \\
\text { 2H-tertaraole }\end{array}$ & & & & & & & & & $2.31 \times 10^{7}$ & 0.22 & & & $2.50 \times 10^{7}$ & 0.10 & & & $1.34 \times 10^{6}$ & \\
\hline
\end{tabular}


Table 1. Cont.

\begin{tabular}{|c|c|c|c|c|c|c|c|c|c|c|c|c|c|c|c|c|c|c|c|}
\hline 28.321 & $\begin{array}{l}\text { (5E)-6,610-dimethylundeca- } \\
\text { 5,9-dien-2-one }\end{array}$ & & & $2.30 \times 10^{7}$ & 0.14 & $4.88 \times 10^{7}$ & 0.28 & $3.09 \times 10^{7}$ & 0.19 & $2.46 \times 10^{7}$ & 0.23 & $3.58 \times 10^{7}$ & 0.22 & $4.22 \times 10^{7}$ & 0.17 & $1.01 \times 10^{7}$ & 0.09 & $1.25 \times 10^{7}$ & $1.12 \times 10^{7}$ \\
\hline 29.385 & 1-(furan-2-ylmethyl)pyrrole & & & $5.54 \times 10^{7}$ & 0.33 & & & & & & & & & & & & & & \\
\hline 30.200 & dodecan-1-ol & & & & & & & & & $3.39 \times 10^{7}$ & 0.32 & & & $4.06 \times 10^{7}$ & 0.16 & & & $4.74 \times 10^{6}$ & \\
\hline 30.590 & Nonatriacontane $<\mathrm{n}->$ & & & $2.50 \times 10^{7}$ & 0.15 & & & & & & & $1.24 \times 10^{7}$ & 0.08 & & & & & & $8.91 \times 10^{6}$ \\
\hline 32.049 & $\begin{array}{l}\text { 2,3,5-trimethylbenzene-1,4- } \\
\text { diol }\end{array}$ & & & $9.42 \times 10^{6}$ & 0.06 & & & & & & & $1.17 \times 10^{7}$ & 0.07 & & & & & & $1.61 \times 10^{6}$ \\
\hline 32.316 & 1-iodohexadecane & & & & & & & & & & & & & & & $5.58 \times 10^{7}$ & 0.50 & & \\
\hline 33.663 & $\begin{array}{l}\text { 1,3,7-trimethylpurine-2,6- } \\
\text { dione }\end{array}$ & & & & & & & & & $2.50 \times 10^{7}$ & 0.23 & & & $3.31 \times 10^{7}$ & 0.13 & & & $5.73 \times 10^{6}$ & \\
\hline 34.795 & $\begin{array}{l}2,3,5 \text {-trimethylbenzene-1,4- } \\
\text { diol }\end{array}$ & & & & & & & & & $4.06 \times 10^{7}$ & 0.38 & & & $1.60 \times 10^{7}$ & 0.06 & & & $1.74 \times 10^{7}$ & \\
\hline 36.303 & 1-iodohexadecane & & & & & & & & & $2.76 \times 10^{7}$ & 0.26 & & & $1.86 \times 10^{7}$ & 0.07 & $1.21 \times 10^{7}$ & 0.11 & $6.36 \times 10^{6}$ & \\
\hline 45.432 & $\begin{array}{l}\text { 1,3,7-trimethylpurine-2,6- } \\
\text { dione }\end{array}$ & $4.34 \times 10^{7}$ & 0.13 & $2.83 \times 10^{7}$ & 0.17 & $1.94 \times 10^{8}$ & 1.10 & $3.89 \times 10^{7}$ & 0.24 & $3.31 \times 10^{7}$ & 0.31 & $1.92 \times 10^{8}$ & 1.18 & $6.62 \times 10^{7}$ & 0.26 & $3.94 \times 10^{7}$ & 0.35 & $7.45 \times 10^{7}$ & $7.84 \times 10^{7}$ \\
\hline тот & & $\begin{array}{l}3.38 \times \\
1010\end{array}$ & 100 & $1.69 \times 10^{7}$ & 100 & $1.77 \times 10^{7}$ & 100 & $1.66 \times 10^{7}$ & 100 & $1.07 \times 10^{7}$ & 100 & $1.63 \times 10^{7}$ & 100 & $2.54 \times 10^{7}$ & 100 & $1.12 \times 10^{7}$ & 100 & & \\
\hline
\end{tabular}

RT = Retention Time; VOCs $=$ Volatile Organic Compounds; Dev.St $=$ Standard Deviation 
Biologico

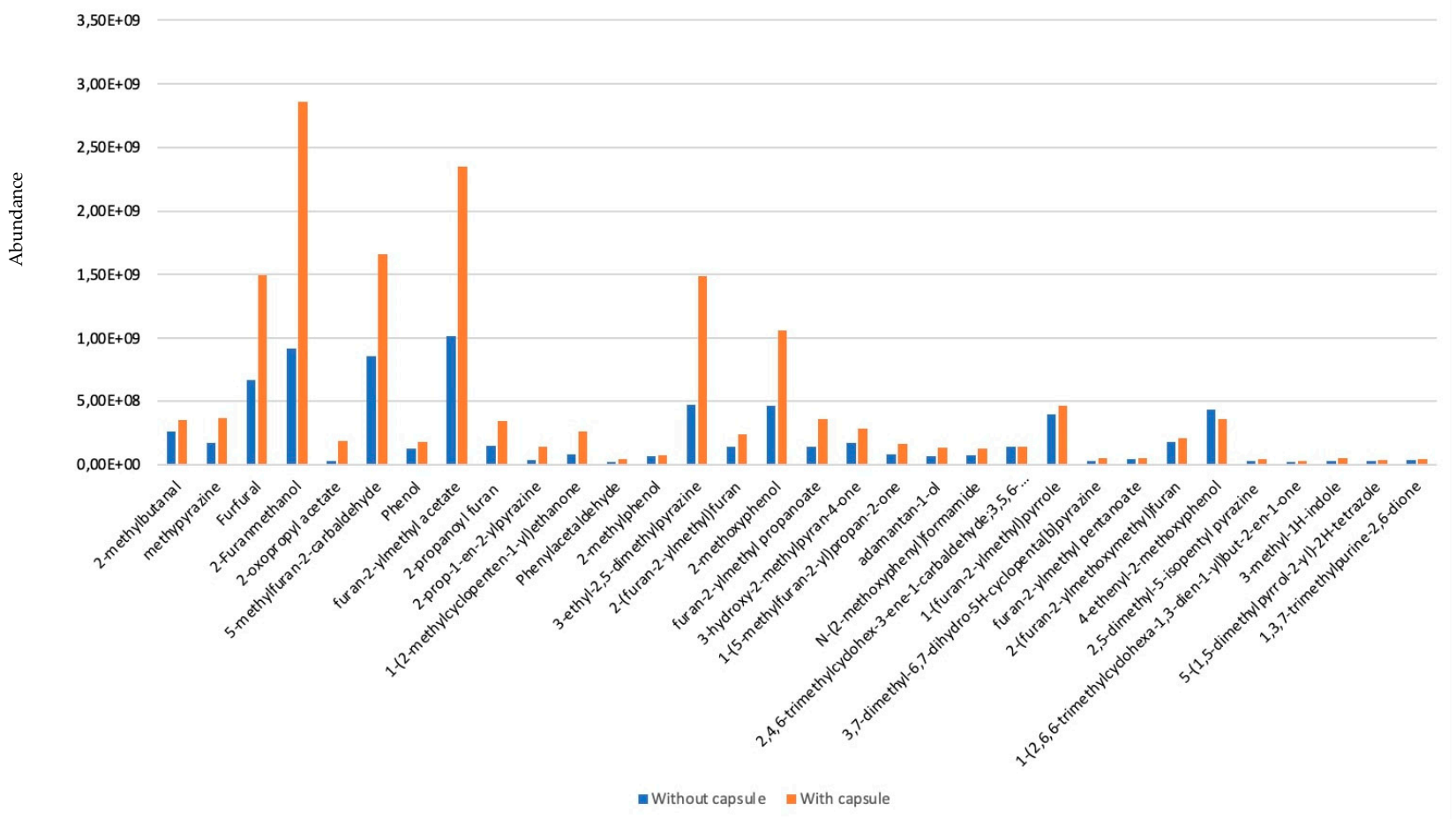

Figure 1. Comparison abundance value of common compound between Biologico coffee "with capsule" and "without capsule".

Deciso

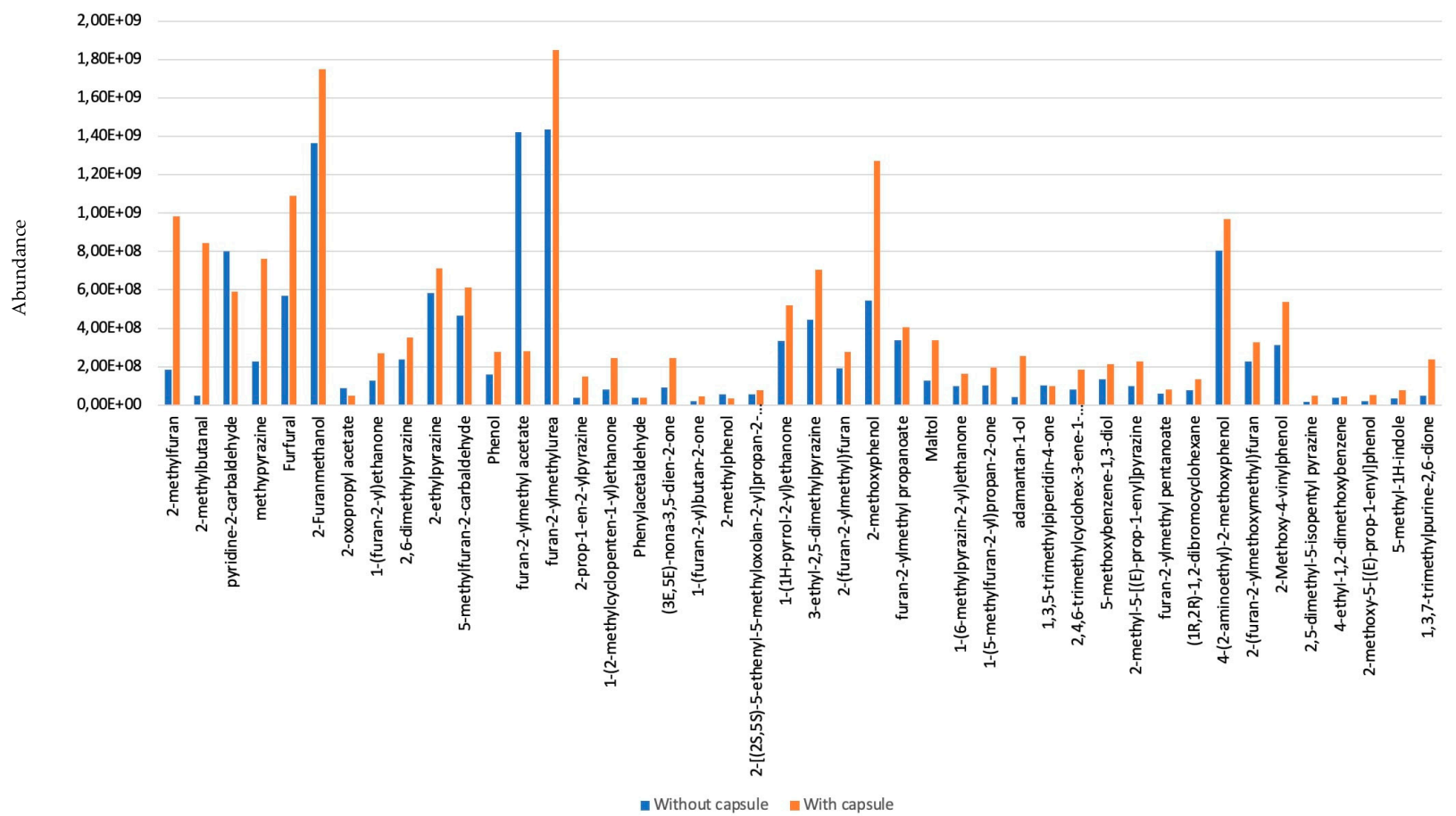

Figure 2. Comparison abundance value of common compound between Deciso coffee "with capsule" and "without capsule". 
Dolce

$2,50 E+09$

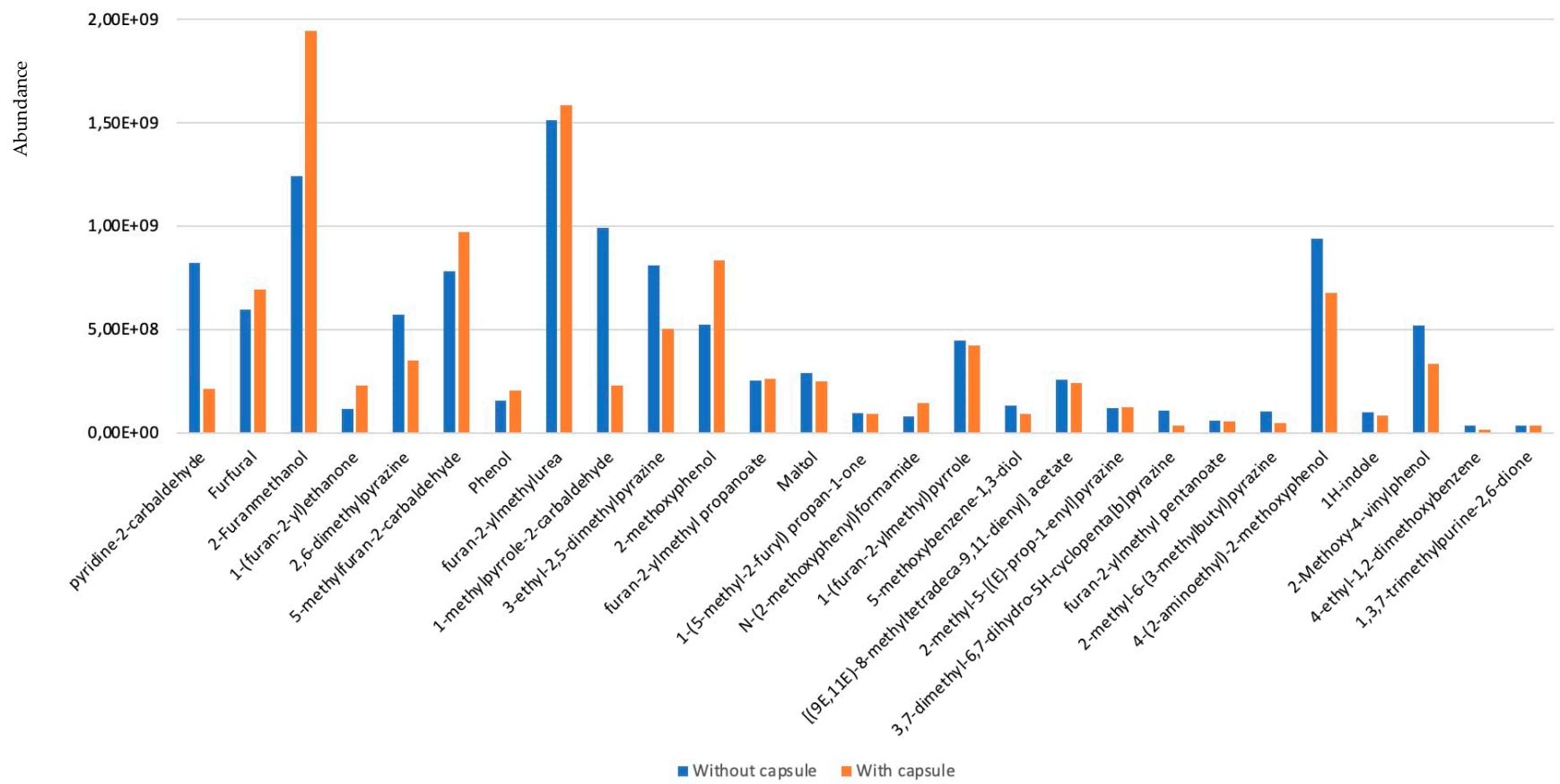

Figure 3. Comparison abundance value of common compound between Dolce coffee "with capsule" and "without capsule".

Guatemala

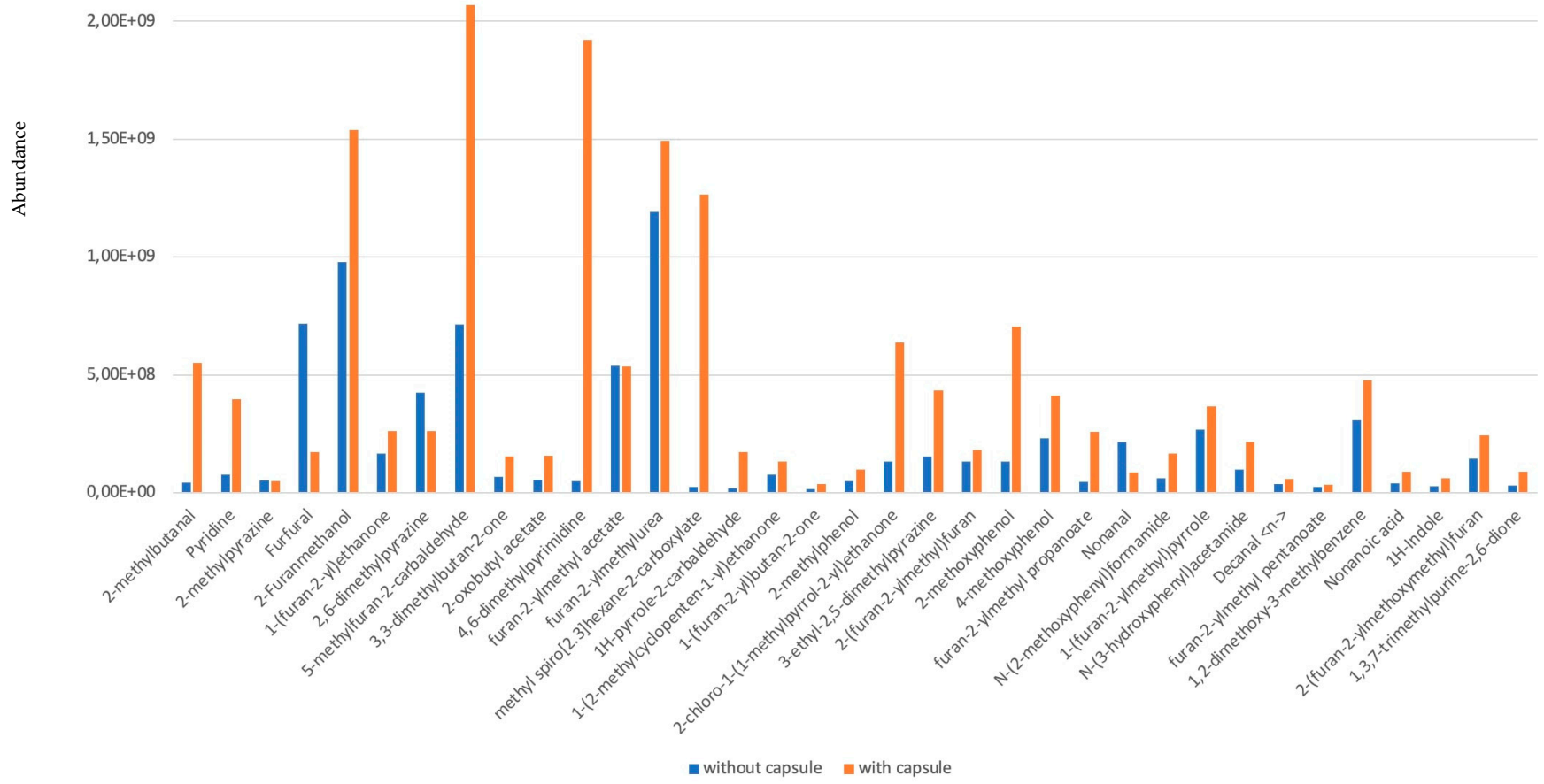

Figure 4. Comparison abundance value of common compound between Guatemala coffee "with capsule" and "without capsule". 
Indeed, over one thousand different chemical entities have been identified in coffee beans; a significant number of these will be extracted during brewing, a comparatively small subset of chemicals impact on the aroma. The coffee aroma intensity is influenced by the compression of the coffee pod, which improves the extraction of the volatile fraction. Indeed, the results show how the coffee obtained by the pods has a more intense volatilome. Studies often consider two main factors when discerning a compounds' aroma impact: the concentration of the compound, and the compound's odor threshold, i.e., the minimum concentration at which we can detect its smell [19]. The ratio of a compound's concentration to its odor threshold gives the compounds an 'odor activity value' (OAV) [20], which gauges its importance to the overall aroma. Several families of compounds are significant contributors to coffee's aroma. Some examples can be families of compounds such as aldehydes, which generally add a fruity and green aroma, furans, which contribute caramellike odors, and pyrazines, which have an earthy scent. Guaiacol and related phenolic compounds offer smoky, spicy tones, and pyrroles and thiophenes are also present in low concentrations. A closer look to all the volatile compounds that we found with the GC-MS results, shown in Table 1, explains how several of them were common concerning the four samples with and without capsule. Regarding the first one, there were 29 volatiles in common between the sample with capsule between a total number of 205 total compounds found. In particular:

- Five of them are aldehydes: 2-methyl- Butanal; 5-methyl-2-furancarboxaldehyde; 1H-Pyrrole-2-carboxaldehyde; Furfural; and Isocyclocitral. Aldehydes are important molecules because they enrich the volatilome with floral flavors [21].

- Three are pyrazines: methyl-Pyrazine; 2,6-dimethyl-Pyrazine; 2-methyl-Pyrazine; 3-ethyl-2,5-dimethyl Pyrazine. Pyrazines are compounds formed during cooking processes in food matrices consisting of both a sugar fraction and a protein/amino acid fraction, characterizing the typical toasted notes that are perceived from the food.

- Six are of them are alcohols 2-Furanmethanol; 1-1(H-pyrazol-1-yl)-2-Propanone; 2Furanmethanol acetate; 2-Furanmethanol, propanoate; Maltol; Benzo-2,3-pyrrole. Alcohols can have two functions. They can directly influence the volatilome or indirectly act as precursors of aldehydes and ketones.

- Three are furans: 2-Hexanoylfuran; 2,2'-methylenebis-Furan; 2,2'-[oxybis(methylene)]bisFuran. Furan is a colorless chemical with a low molecular weight and highly volatile. It forms as a result of food heating and contributes to the roasted taste in some. The coffee and in particular when roasted, represents a source of alimentary exposure to the furan.

- $\quad$ Five are ketones: 1-(2-methyl-1-cyclopenten-1-yl)-Ethanone; 1-(6-Methyl-2-pyrazinyl)1-ethanone; 1-(5-methyl-2-furanyl)-1-Propanone; 1-(2,5-dihydroxyphenyl)-Ethanone; 1-(2,6,6-trimethyl-1,3-cyclohexadien-1-yl)-(E)- 2-Buten-1-one. Ketones are important molecules because they enrich the volatilome with floral flavors.

- Two are phenols: Phenol; 2-methoxy-Phenol. Phenols are molecules possible to find in the raw material. Generally, polyphenols and chlorogenic acid have higher concentration.

- Two acids: Propanoic acid ethenyl ester; Furfuryl pentanoate. Carboxylic acids are a part of the non-volatile component of coffee seeds after roasting, generated as a result of complex reactions that occur during roasting.

- One alkaloid: Caffeine. This compound is responsible for the bitterness.

On the other hand, the results about coffee obtained without capsule, shown in Table 1, highlight a few more common compounds between the four samples, in particular 34:

- Six of them are aldehydes: 2-methyl-Butanal; Furfural; Nonanal; Phenylacetaldehyde; 1-methyl-1H-Pyrrole-2-carboxaldehyde.

- $\quad$ Five are pyrazines: methyl-Pyrazine; 2,6-dimethyl-Pyrazine; 3-ethyl-2,5-dimethyl Pyrazine; (1-methylethenyl)-Pyrazine; 3,5-diethyl-2-methyl-) Pyrazine.

- Six of them are alcohols: (2-Furanmethanol; 2-Furanmethanol acetate; Maltol; 1-(2furanylmethyl)-1H-Pyrrole; Indole; 5-methyl-1H-Indole. 
- Three are furans: 2,2'-methylenebis-Furan; 5-methyl-2-furancarboxaldehyde; N-(2furfuryl)-Urea.

- $\quad$ Five are ketones: 1-(2-methyl-1-cyclopenten-1-yl)-Ethanone; 1-(5-methyl-2-furanyl)-1Propanone; 1-(acetyloxy)-2-Propanone; 1-(2-furanyl)-Ethanone; 1-(2-furanyl)-2-Butanone; 1-(1H-pyrrol-2-yl)-) Ethanone.

- Five are phenols: Phenol; 2-methoxy- Phenol; 2-methyl-Phenol; 2-methoxy-5-(1propenyl)-, (E)-Phenol; <4-vinyl-> Guaiacol.

- Two acids: Furfuryl pentanoate; Furfuryl propionate.

- One Benzene: 4-ethyl-1,2-dimethoxy Benzene.

- One Alkaloid: Caffeine.

Overall, it is clear how all the samples are similar and consequently how the packaging protection does not cause a significant change on the volatiles detected, but only has an influence in the abundance level of several of them. This result was validated using the statistical analysis of ANOVA and T of Tukey in order to verify which samples were statistically different between each other. The ANOVA test was performed on all the compounds for each sample with and without capsule. ANOVA results showed a $p$-value of 0.0017 which indicates that there are differences between the various types of samples. To verify which extracted coffees were different from each other Tukey test showed that the only significant differences statistically significant are between Biologico (capsule) - Guatemala (no capsule) and Guatemala (capsule)-Guatemala (no capsule) with a $p$ value of 0.0032 and 0.0289 , respectively.

Regarding PCA analysis the results are shown in Figures 5-8. In all four cases the comparison was made between the volatilome of the sample with and without capsule.

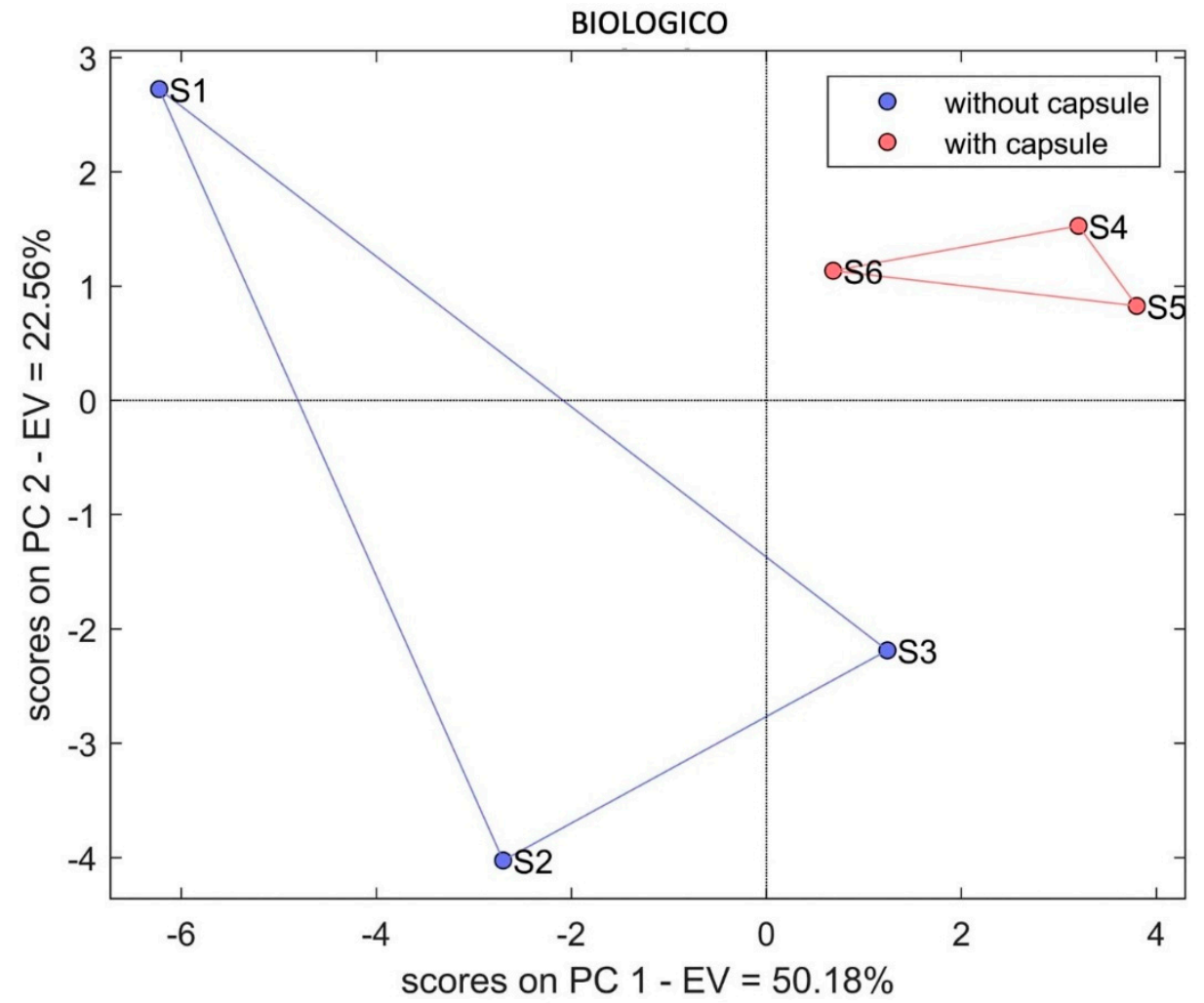

Figure 5. PCA score showing the comparison between volatile spectra compound of Biologico coffee "with capsule" and "without capsule" exhibiting an EV (explained variance) $=72.74 \%$. 


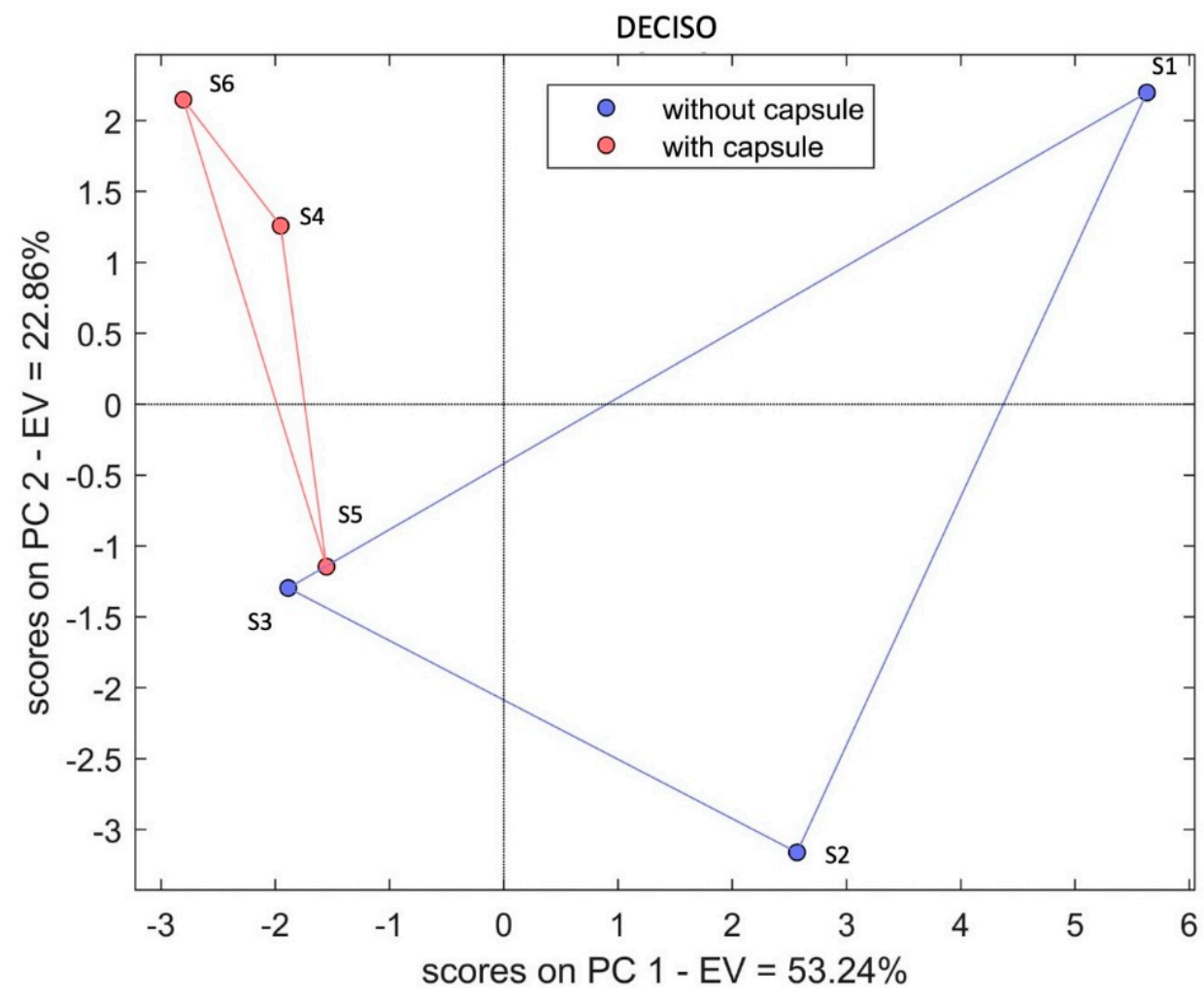

Figure 6. PCA score showing the comparison between volatile spectra compound of Deciso coffee "with capsule" and "without capsule" exhibiting an EV (explained variance) $=76.10 \%$.

DOLCE

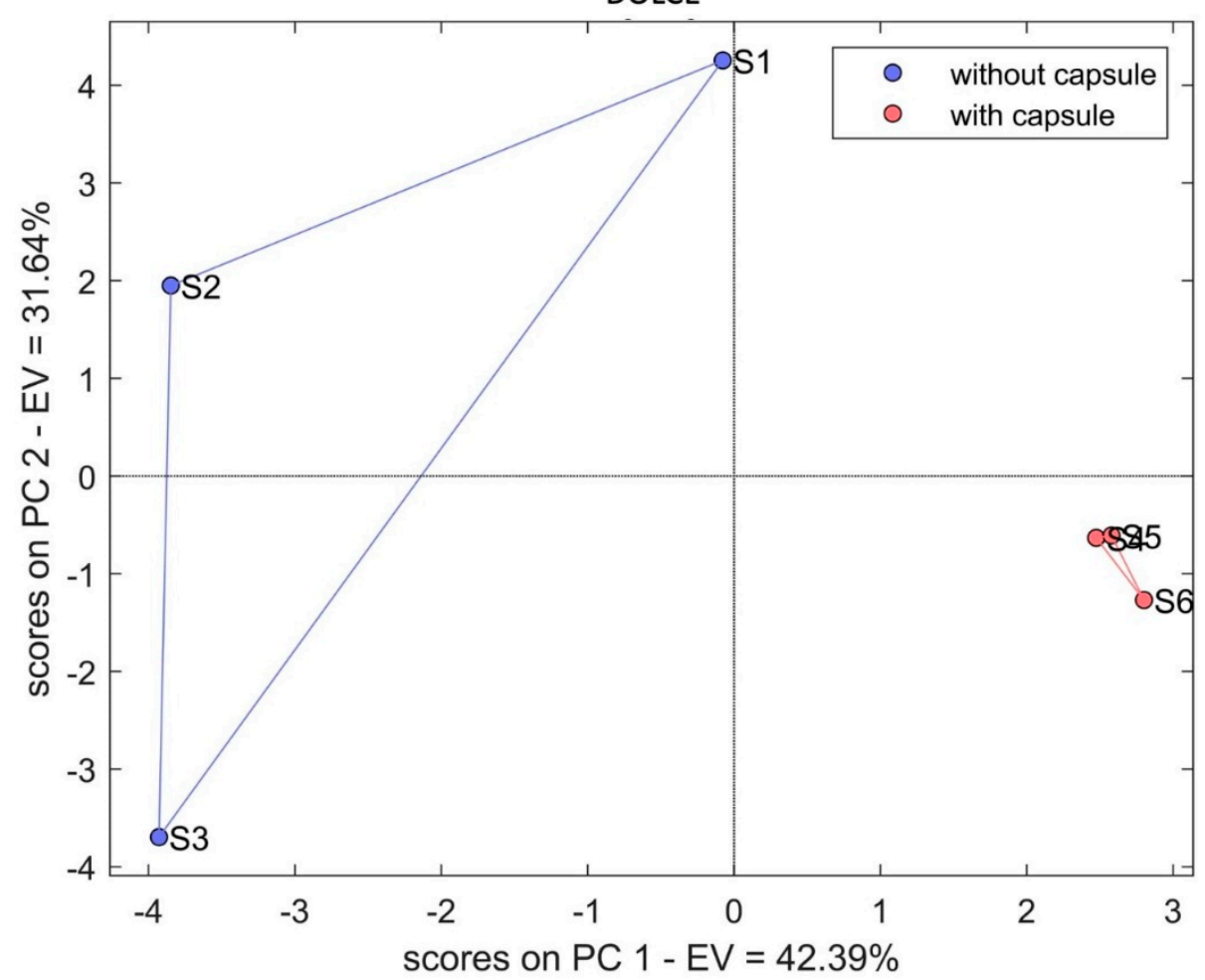

Figure 7. PCA score showing the comparison between volatile spectra compound of Dolce coffee "with capsule" and "without capsule" exhibiting an EV (explained variance) $=74.03 \%$. 


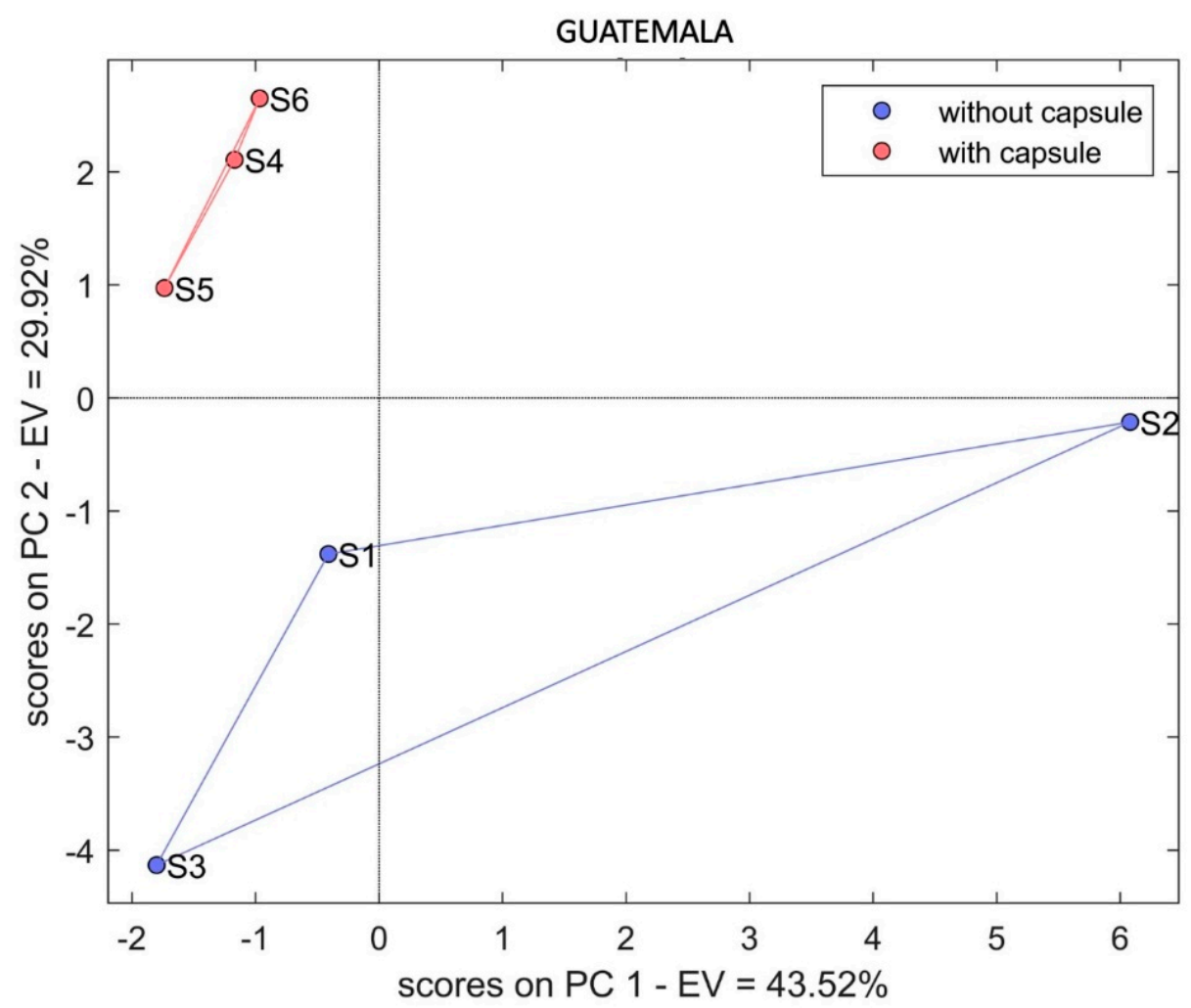

Figure 8. PCA score showing the comparison between volatile spectra compound of Guatemala coffee "with capsule" and "without capsule" exhibiting an EV (explained variance) $=73.44 \%$.

We can conclude overall that PCA previously showed the EV was never under the $72 \%$. This represents an optimum result since at least the $72 \%$ of the total variability of the samples was enclosed between the hyperplane (enclosed between the first two principal components), PC1 always being the component with a larger load, reaching $53 \%$ in the case of the Deciso samples. As a matter of fact, the PCA shown in Figures 5-8 confirm what was previously discussed. It is clear how the three replicates for all the four samples extracted with capsule create a compact cluster (S4, S5, S6), separated on the hyperplane compared with that formed by the ones without capsule (S1, S2, S3). In addition, PCA analysis confirms another aspect of this study. Indeed, the coffee capsule clusters are more compact on the hyperplane than the coffee without capsule, meaning that the first ones (S4, $\mathrm{S} 5, \mathrm{S6})$ are more similar due to the performed product standardization.

\section{Conclusions}

This investigation was based on the analysis of four different samples (Biologico, Deciso, Dolce, Guatemala). The results were performed using gas chromatography and mass spectrometry (GC-MS), obtaining the volatilome of the four typologies with and without capsules and allowing to discriminate these two methodic. The multivariate statistics performed confirm the aim of the work. It can be concluded that the coffee aroma intensity is influenced by the presence of packaging. Thus, apart from being able to reduce the migration of oxygen, it allows to decrease the loss of volatile compounds. Indeed, the most important physical and chemical events involved in coffee without capsules during storage are volatile release, oxidation reactions, carbon dioxide release and surface oil migration. These reactions cause the loss of freshness of roasted coffee, which would thus determine a progressive deterioration of the aroma profile that has more impact in ground coffee, and would be reduced with the utilization of the polypropylene capsule. In addition, the economical side is determinant and characteristic as time optimization or standardization are important for any seller, that could also be achieved with the use 
of coffee capsules. Furthermore, coffee capsules can exhibit an extended shelf life of the product and reduce costs of a Ho. Re. Ca. activity. Thus, the professional machines could provide standardized organoleptic characteristics of each coffee, without the need of training the operator. Consequently, coffee capsules can assure a maintenance of the aroma intensity and avoid the deterioration that would occur for the coffee without capsule.

Author Contributions: Conceptualization, G.G., E.N.-C., M.A. and V.S.; methodology, G.G., E.N.-C., M.A. and V.S.; software, G.G. and M.A.; validation, G.G., E.N.-C., M.A. and V.S.; formal analysis, G.G., E.N.-C., M.A. and V.S.; investigation, G.G., E.N.-C., M.A. and V.S.; resources, G.G., E.N.-C., M.A. and V.S.; data curation, G.G., E.N.-C., M.A. and V.S.; writing-original draft preparation, G.G., E.N.-C., M.A. and V.S.; writing-review and editing, G.G., E.N.-C., M.A., P.F. and V.S.; visualization, G.G., E.N.-C., M.A. and V.S.; supervision, G.G., E.N.-C., M.A. and V.S.; project administration, G.G., E.N.-C., M.A. and V.S.; funding acquisition, E.N.-C., M.A. and V.S. All authors have read and agreed to the published version of the manuscript.

Funding: This research received no external funding.

Institutional Review Board Statement: Not applicable.

Informed Consent Statement: Not applicable.

Acknowledgments: Authors wants to thank "Caffè Molinari" (Via Francia, Modena, Italy) for the gently sample supply.

Conflicts of Interest: The authors declare no conflict of interest.

\section{References}

1. Robertson, G.L. Food Packaging and Shelf Life: A Practical Guide; CRC Press: Boca Raton, FL, USA, 2009 ; pp. $199-214$.

2. Bates, R.H.; Greif, A.; Levi, M.; Rosenthal, J.L.; Weingast, B.R. Analytic Narratives; Princeton University Press: Princeton, NJ, USA, 2020; 264p.

3. Arabica e Robusta: Per una Miscela Perfetta Serve Equilibrio. Available online: https://www.dolce-gusto.it/mydolcegusto/ tutto-sul-caffe/arabica-robusta-miscela / (accessed on 21 June 2020).

4. Favati, F.; Condelli, N.; Galgano, F.; Caruso, M.C. Extra virgin olive oil bitterness evaluation by sensory and chemical analyses. Food Chem. 2013, 139, 949-954. [CrossRef] [PubMed]

5. Qualcosa da Sapere Sulle Capsule del Caffè. 2019. Available online: http://www.tbspa.it/qualcosa-da-sapere-sulle-capsule-del-caffe/ (accessed on 15 July 2021).

6. $\quad$ Otoukesh, M.; Vera, P.; Wrona, M.; Nerin, C.; Es'Haghi, Z. Migration of dihydroxyalkylamines from polypropylene coffee capsules to Tenax ${ }^{\circledR}$ and coffee by salt-assisted liquid-liquid extraction and liquid chromatography-mass spectrometry. Food Chem. 2020, 321, 126720. [CrossRef] [PubMed]

7. Nicoli, M.C.; Calligaris, S.; Manzocco, L. Shelf-Life Testing of Coffee and Related Products: Uncertainties, Pitfalls, and Perspectives. Food Eng. Rev. 2009, 1, 159-168. [CrossRef]

8. Najar, B.; Nardi, V.; Cervelli, C.; Pistelli, L. Volatiloma analyses of four South African Helichrysum spp. grown in Italy. Nat. Prod. Res. 2020. Epub ahead of print. [CrossRef] [PubMed]

9. Lehotay, S.J.; Hajslova, J. Application of gas chromatography in food analysis. TrAC Trends Anal. Chem. 2002, 21, 686-697. [CrossRef]

10. Núñez-Carmona, E.; Abbatangelo, M.; Sberveglieri, V. Internet of Food (IoF), Tailor-Made Metal Oxide Gas Sensors to Sup-port Tea Supply Chain. Sensors 2021, 21, 4266. [CrossRef] [PubMed]

11. Navarini, L.; Rivetti, D. Water quality for Espresso coffee. Food Chem. 2010, 122, 424-428. [CrossRef]

12. Chen, P.H.; Richardson, S.D.; Krasner, S.W.; Majetich, G.; Glish, G.L. Hydrogen Abstraction and Decomposition of gmBromopicrin and Other Trihalogenated Disinfection Byproducts by GC/MSPaul H. Glish Environ. Sci. Technol. 2002, 36, 3362-3371. [CrossRef] [PubMed]

13. Núñez-Carmona, E.; Abbatangelo, M.; Sberveglieri, V. Innovative Sensor Approach to Follow Campylobacter jejuni Devel-opment. Biosensors 2019, 9, 8. [CrossRef] [PubMed]

14. Abbatangelo, M.; Nunez-Carmona, E.; Sberveglieri, V. Novel Equipment for Food Quality Control: An Iot Nanowire Gas Sensors Array. Chem. Eng. Trans. 2019, 75, 25-30.

15. Zanin, R.C.; Smrke, S.; Kurozawa, L.E.; Yamashita, F.; Yeretzian, C. Modulation of aroma release of instant coffees through microparticles of roasted coffee oil. Food Chem. 2021, 341, 128193. [CrossRef]

16. Eichelberger, J.W.; Harris, L.E.; Budde, W.L. Reference Compound to Calibrate Ion Abundance Measurements in Gas Chromatography-Mass Spectrometry Systems. Anal. Chem. 1975, 47, 995-1000. [CrossRef] 
17. Caprioli, G.; Cortese, M.; Cristalli, G.; Maggi, F.; Odello, L.; Ricciutelli, M.; Sagratini, G.; Sirocchi, V.; Tomassoni, G.; Vittori, S. Optimization of espresso machine parameters through the analysis of coffee odorants by HS-SPME-GC/MS. Food Chem. 2012, 135, 1127-1133. [CrossRef] [PubMed]

18. Crozier, T.W.M.; Stalmach, A.; Lean, M.E.J.; Crozier, A. Espresso coffees, caffeine and chlorogenic acid intake: Potential health implications. Food Funct. 2012, 3, 30-33. [CrossRef] [PubMed]

19. The Chemical Compounds behind the Aroma of Coffee. 2015. Available online: https://www.compoundchem.com/2015/02/17 / coffee-aroma/ (accessed on 18 July 2021).

20. Wu, C.; Liu, J.; Yan, L.; Chen, H.; Shao, H.; Meng, T. Assessment of odor activity value coefficient and odor contribution based on binary interaction effects in waste disposal plant. Atmos. Environ. 2015, 103, 231-237. [CrossRef]

21. Rao, N.Z.; Fuller, M.; Grim, M.D. Physiochemical Characteristics of Hot and Cold Brew Coffee Chemistry: The Effects of Roast Level and Brewing Temperature on Compound Extraction. Foods 2020, 9, 902. [CrossRef] [PubMed] 